\title{
Neural Network-Based Sensitivity Analysis of Summertime Convection over the Continental United States
}

\author{
FILIPE AIRES \\ Estellus, and Laboratoire de l'Etude du Rayonnement et de la Matière en Astrophysique, CNRS, Observatoire de Paris, \\ Paris, France, and Department of Earth and Environmental Engineering, Columbia University, New York, New York \\ PIERRE GENTINE \\ Department of Earth and Environmental Engineering, Columbia University, New York, New York
}

KIRSTEN L. FINDELL

Geophysical Fluid Dynamics Laboratory, Princeton, New Jersey

BENJAMIN R. LINTNER

Rutgers, The State University of New Jersey, New Brunswick, New Jersey

CHRISTOPHER KERR

University Corporation for Atmospheric Research/GFDL, Princeton, New Jersey

(Manuscript received 27 February 2013, in final form 9 November 2013)

\begin{abstract}
Although land-atmosphere coupling is thought to play a role in shaping the mean climate and its variability, it remains difficult to quantify precisely. The present study aims to isolate relationships between early morning surface turbulent fluxes partitioning [i.e., evaporative fraction (EF)] and subsequent afternoon convective precipitation frequency and intensity. A general approach involving statistical relationships among input and output variables, known as sensitivity analysis (SA), is used to develop a reduced complexity metamodel of the linkage between EF and convective precipitation. Two additional quantities characterizing the early morning convective environment, convective triggering potential (CTP) and low-level humidity (HIlow) deficit, are included. The SA approach is applied to the North American Regional Reanalysis (NARR) for June-August (JJA) conditions over the entire continental United States, Mexico, and Central America domain. Five land-atmosphere coupling regimes are objectively characterized based on CTP, HIlow, and EF. Two western regimes are largely atmospherically controlled, with a positive link to CTP and a negative link to HIlow. The other three regimes occupy Mexico and the eastern half of the domain and show positive links to EF and negative links to HIlow, suggesting that both surface fluxes and atmospheric humidity play a role in the triggering of rainfall in these regions. The regimes associated with high mean EF also tend to have high sensitivity of rainfall frequency to variations in EF. While these results may be sensitive to the choice of dataset, the approach can be applied across observational, reanalysis, and model datasets and thus represents a potentially powerful tool for intercomparison and validation as well as for characterizing land-atmosphere interaction regimes.
\end{abstract}

\section{Introduction}

Sensitivity analysis (SA) is a branch of statistics applied to the study of complex models. Its main purpose

Corresponding author address: F. Aires, Estellus, LERMA Observatoire de Paris, 61 avenue de l'Observatoire, 74014 Paris, France. E-mail: filipe.aires@estellus.fr is the estimation of model output-to-input sensitivities. SA has been widely applied in a diverse range of fields including social sciences, engineering, economics, and geophysical sciences for research prioritization (e.g., to identify which parameters warrant further investigation or validation through measurements); for model synthesis to aid in comprehension of complex models; or for system investigation (e.g., to identify which 
regions of input space render the system most unstable to perturbations).

For a multidimensional, strongly coupled, nonlinear system like the climate system, estimates of output-toinput sensitivities are clearly desirable not only from an operational standpoint (e.g., prediction) but from a theoretical one as well. State-of-the-art climate models include dozens of 3D fields on spatial resolutions of tens to hundreds of kilometers and temporal resolutions of hours. Given the overall complexity of such models, not to mention the real climate system, complete understanding of climate system processes is often limited, especially when multiple interacting processes are involved. Methodologies that reduce complexity can provide powerful insights into climate processes. In this regard, SA may facilitate analysis of the climate system or subsystem thereof by employing simpler and faster statistical models known as metamodels. A metamodel isolates the most important variables in a combination of physical processes. It will be seen how various combinations of inputs can be tested by the metamodel to find the variables that most strongly impact convective precipitation. Moreover, because a metamodel can be calibrated against real observations or climate model outputs, it can be used for process-level evaluation of models.

In the present study, we apply SA to the study of landatmosphere coupling. As broadly understood, landatmosphere coupling encompasses the interactions and processes (radiation, turbulence, hydrology, biogeochemistry) linking the land surface and overlying atmosphere; for extensive reviews, see Seneviratne et al. (2010) and Betts and Silva Dias (2010). One aspect of land-atmosphere coupling that has received significant attention involves potential feedbacks between precipitation and soil moisture; as such feedbacks are expected to impact the variability of climate across multiple time scales and to modulate the persistence and intensity of droughts and the occurrence of extreme events. However, while the processes involved in land-atmosphere coupling are expected to influence mean climate and its variability, isolating unambiguous signatures of this coupling in both observations and models has proved difficult. Among the key challenges are the spatial heterogeneity and scale dependence of the processes involved.

Our objective here is to isolate the relationships between early morning surface turbulent flux partitioning, represented in terms of the evaporative fraction (EF; i.e., the ratio of latent heat flux to the sum of sensible and latent heat fluxes), and subsequent (same day) convective precipitation frequency and intensity. Previous studies, notably Findell and Eltahir (2003a,b) and Findell et al. (2011), have examined aspects of this coupling using in situ radiosonde data and the North American Regional
Reanalysis (NARR) data in a relatively simple statistical framework Mesinger et al. (2006). In what follows, we demonstrate the utility of SA for diagnosing the EFconvective precipitation relationships described in Findell et al. (2011). The analysis is performed using the same data as in Findell et al. (2011), although the methodology presented can be used with any kind of model or observational products.

The remainder of this paper is organized as follows. A detailed overview of the SA methodology is provided in appendix 5. A review of land-atmosphere coupling over the continental United States (CONUS) is given in section 2 together with a first analysis of the datasets used in this paper. Results of the analysis are presented in section 3. Finally, conclusions and perspectives are presented in section 4 .

\section{Land-atmosphere coupling over CONUS}

\section{a. Introduction}

The land surface and the overlying atmosphere interact through a set of coupled energy and water cycle feedback processes (Brubaker and Entekhabi 1996; Betts et al. 1996; Koster et al. 2004; Santanello et al. 2007). The coupling between the land surface and the atmosphere is mediated by the state of the surface, which modifies the partitioning of both the surface energy and water budgets (Koster and Suarez 1994; Milly and Dunne 1994; Robock et al. 1995; Salvucci 2001; Seneviratne et al. 2006, 2010) and over time scales ranging from minutes to interannual, and spatial scales ranging from millimeters to hundreds of kilometers (Pielke et al. 1998; Katul et al. 2012). In turn, the surface energy and water budgets affect the state of the overlying atmosphere, including near-surface turbulence, thermodynamic profiles, stability, clouds, precipitation, and dynamics (Pan and Mahrt 1987; Ek and Holtslag 2004; Gentine et al. 2007; Seneviratne et al. 2010; Findell et al. 2011; Gentine et al. 2013a). Changes in the atmospheric state, especially those within the planetary boundary layer (PBL; Santanello et al. 2005, 2007, 2009, 2011), can further feed back onto the surface across different spatial and temporal scales (Brubaker and Entekhabi 1996; Gentine et al. 2010).

How soil moisture ultimately affects precipitation has important consequences for interpreting and predicting variability in the real climate system (Koster et al. 2003; Guo et al. 2012) as well as in models (Koster et al. 2004; Dirmeyer et al. 2006; Guo et al. 2006; Koster et al. 2006; Seneviratne et al. 2006; Koster and Mahanama 2012). For example, the occurrence of positive feedbacks between anomalous soil moisture conditions and subsequent precipitation may contribute to the persistence of extreme 
drought conditions and heat waves (Entekhabi et al. 1992; Schär and Jendritzky 2004; Schär et al. 2004; Seneviratne et al. 2006). Moreover, synoptic and seasonal forecasts have been shown to be extremely sensitive to soil moisture initialization (Sutton et al. 2006; Guo et al. 2012). Quantitative assessment of the impacts of large-scale deforestation, land use modification, and anthropogenic climate change requires detailed mechanistic understanding of the soil moisture-precipitation feedback (Notaro 2008). Soil moisture is currently thought to impact precipitation in the following ways: (i) local evaporative recycling (Eltahir 1989; Savenije 1995; Dirmeyer et al. 2009), (ii) large-scale advection and/or convergence of atmospheric moisture (Pal and Eltahir 2003; Cook et al. 2006; Taylor 2008; Lintner and Neelin 2009; Hohenegger et al. 2009; Lintner et al. 2013), and (iii) modification of the properties of the PBL (Betts et al. 1996; Pal and Eltahir 2001; Findell and Eltahir 2003a; Gentine et al. 2013b). Methods (i) and (ii) may be related through considerations of scale: while local evaporative recycling is assumed to occur over a sufficiently large region such that any evaporated moisture ultimately remains in that region, for smaller regions of interest the nonlocal horizontal advective transport processes may become dominant.

For (iii), two mechanisms are thought to operate at daily time scales. The first mechanism is related to lowering of PBL height and increasing PBL moist static energy (MSE) in the presence of positive soil moisture anomalies (Betts et al. 1996; Pal and Eltahir 2001; Findell and Eltahir 2003a; Gentine et al. 2013b). That is, a positive soil moisture anomaly leads to an increase of latent heat flux (and a decrease of sensible heat flux), thereby reducing the atmospheric boundary layer (ABL) height and increasing MSE (Gentine et al. 2013b). In fact, MSE per unit PBL volume may increase more strongly than MSE itself because of PBL lowering. Additionally, the level of free convection (LFC) and lifting condensation level (LCL) are lowered toward the PBL top, leading to an increased potential for convective development. Attendant column radiative changes can, in turn, modulate the strength of this mechanism (Pal and Eltahir 2001), as can the coupling of the lower free troposphere and PBL (Margulis and Entekhabi 2001). In total, this mechanism is expected to generate a positive precipitation-soil moisture feedback. The second mechanism is associated with triggering convection by raising the PBL height toward the LFC over deep-PBL regions (e.g., the Sahel) (Gentine et al. 2013b) or through induced local or mesoscale circulations (Taylor et al. 2011, 2012). Since dry surfaces are associated with flux partitioning favoring sensible heat flux and increased buoyancy, this pathway corresponds to a negative feedback of soil moisture on precipitation. This is thought to occur mostly under hot, unstable conditions (Westra et al. 2012), regions with an upperlevel moisture source (Findell and Eltahir 2003a), and deep boundary layers (Gentine et al. 2013b).

\section{b. Land-atmosphere coupling metrics}

One of the key challenges in the study of landatmosphere coupling is the development of straightforward metrics for quantifying the strength of the coupling in observations and models (Sun and Wang 2012). The Global Land-Atmosphere Coupling Experiment (GLACE; Koster et al. 2004; Dirmeyer et al. 2006; Notaro 2008) introduced a well-known metric based on the intra-ensemble spread of variance in interactive and prescribed soil moisture simulations, although this metric cannot be obtained directly from observations. Another widely used approach involves the estimation of recycling (Brubaker et al. 1993; Eltahir and Bras 1996; Schär et al. 1999; Dominguez et al. 2006; Dirmeyer and Brubaker 2007), such as the fraction of recycled to total precipitation occurring over a region, although such estimates may depend on the details of the area and scale considered and may be difficult to generalize (van der Ent and Savenije 2011). Another approach consists in the use of mixing diagrams (Berg and Stull 2004; Santanello et al. 2005, 2007, 2009, 2011) indicating the modification of the PBL by surface heating and moistening, entrainment at the boundary layer top, and advection. Interesting new metrics have recently been developed. For instance, Dirmeyer et al. (2006) use an index of the sensitivity of surface fluxes to soil moisture variations. In Mei and Wang (2012), the probability density function of conditioned correlation between soil moisture and subsequent precipitation or surface temperature is defined as a metric for the coupling strength. Wei and Dirmeyer (2012) quantify both locally and remotely the evapotranspiration-precipitation using a backtrajectory method for water transport. In Zeng et al. (2010), a new parameter $\Gamma$ is proposed to estimate the land-precipitation coupling strength based on the ratio of the covariance between monthly or seasonal precipitation and evaporation anomalies over the variance of precipitation anomalies.

Findell and Eltahir (2003a,b) developed a framework for determining when land surface conditions are likely to influence subsequent convection utilizing the convective triggering potential (CTP) as a proxy for the lower free troposphere stability and the low-level humidity index (HIlow) as a proxy for the humidity deficit of the low-level air. These variables have been used to assess the large-scale potential for convective precipitation and to quantify the soil moisture-precipitation feedback on a large scale (Findell et al. 2011; Tuinenburg et al. 2011; Ferguson and Wood 2011). While CTP is related 
to the convective available potential energy (CAPE), its restriction to the lower free troposphere is thought to reflect the vertical environment with which the developing boundary layer initially interacts (Findell and Eltahir 2003a,b). Findell et al. (2011) show that high evaporative fraction (i.e., the fraction of available energy consumed as latent heat flux) enhances the probability of afternoon rainfall east of the Mississippi and in Mexico during the summer months using the NARR dataset. They further demonstrate that EF has a negligible impact on the magnitude of a convective rainfall event. To evaluate the change in the frequency and quantity of convective rainfall induced by a change in EF, Findell et al. (2011) introduced the triggering feedback strength (TFS) and amplification feedback strength (AFS) metrics. These metrics are based on sensitivities to EF but are complex to estimate and may be difficult to generalize. In particular, it might be difficult to introduce other potentially important factors in the analysis since the estimation of the sensitivities using more than three variables is technically challenging. It will be seen in the following that SA offers a convenient alternative from which more general relationships, unambiguous sensitivities, and potential dependencies on more variables can be derived. Furthermore, the SA results will be shown to be consistent with the TFS and AFS metrics.

\section{c. Datasets}

NARR assimilates atmospheric profiles (temperature, water vapor, wind) from rawinsondes and dropsondes, in situ measurements, and satellite observations. NARR offers two advantages over earlier global reanalysis datasets: finer resolution (approximately 30-km grid spacing) and the ingestion of hourly precipitation and near-surface humidity. NARR has been shown to be more reliable than many other reanalysis products for hydrometeorological studies (Mesinger et al. 2006; Mitchell et al. 2004). Twenty-five years of hourly data are available from 1979 to 2003. Only June-August (JJA) data are included in the evaluation of the feedback since convective rainfall and land-atmosphere feedbacks are believed to be less important during winter. The spatial domain of the analysis is North America and Mexico; locations north of $50^{\circ}$ latitude are excluded because of the lack of hourly precipitation data available for assimilation into the NARR (Findell et al. 2011).

Figure 1 depicts June 1979 means and standard deviations of CTP, HIlow, EF, and $R$ (rainfall) maps. Let us first consider the convective triggering potential (CTP), defined by Findell and Eltahir (2003a) as the area between the observed temperature profile and a moist adiabat originating at the observed temperature $100 \mathrm{hPa}$ above the surface and extending to $300 \mathrm{hPa}$ above the surface. When the lapse rate is close to dry adiabatic, CTP is large and areas of high sensible heat flux may have an advantage in triggering convection. Smaller but still positive CTP means that the lapse rate is closer to moist adiabatic, giving areas of high latent heat flux a convective advantage. Negative CTP indicates a temperature inversion that inhibits locally driven deep convection over the land or mesoscale-synoptic influence on convection triggering. HIlow is the sum of the dewpoint depression ( $T-T_{d}$, where $T$ is air temperature and $T_{d}$ is dewpoint temperature) at 50 and $150 \mathrm{hPa}$ above the ground surface. Findell and Eltahir (2003a,b) showed that when the atmosphere was excessively dry (HIlow $>15 \mathrm{~K}$ ), rainfall in the model was prohibited by the atmospheric conditions but when this deficit was small $(<5 \mathrm{~K})$, the atmosphere was so close to saturation that moist convection becomes likely over any surface. Flux partitioning at the land surface is defined using EF, which is reasonably constant during daylight hours (Crago 1996; Crago and Brutsaert 1996; Gentine et al. 2011, 2007). Findell and Eltahir (2003b) showed that in drier atmospheres high sensible heat flux is a good trigger of convection while in more humid atmospheres large contributions of humidity from the land surface was a more effective trigger. Findell et al. (2011) and Berg et al. (2013) extended this result to show that the impact of flux partitioning is largely felt in the triggering of rainfall events rather than in an enhancement of rainfall amounts once convection is initiated. In this study, CTP and HIlow are evaluated using the 6 to 9 a.m. average, $\mathrm{EF}$ is evaluated from 9 a.m. to noon, and rainfall is considered from noon to 6 p.m., following Findell et al. (2011). It should be noted that in the mean CTP or STD of HIlow maps of Fig. 1, an artifact longitudinal anomaly can be seen at $-112.5^{\circ} \mathrm{W}$. This is due to the adjustment of 3-hourly data to local times. In the central portion of each 3-h time zone block the morning window is indeed 6-9 a.m., while in the western third the morning window is $1 \mathrm{~h}$ earlier (5-8 a.m.), and in the eastern third the morning window is $1 \mathrm{~h}$ later (7-10 a.m.). This means that at the longitudinal interfaces between 3-h time blocks, the 7-10 a.m. section from one block is adjacent to the 5-8 a.m. section from another. This produces the artifact seen at $-112.5^{\circ} \mathrm{W}$.

Some filtering of the dataset is needed in order to focus the analysis toward local processes and avoid, as much as possible, the effects of the large-scale circulation. Our filtering follows Findell et al. (2011). First, only daily data points without early morning rainfall between 6 a.m. and noon are considered, in order to limit the impact of long duration rainfall events. Second, data 

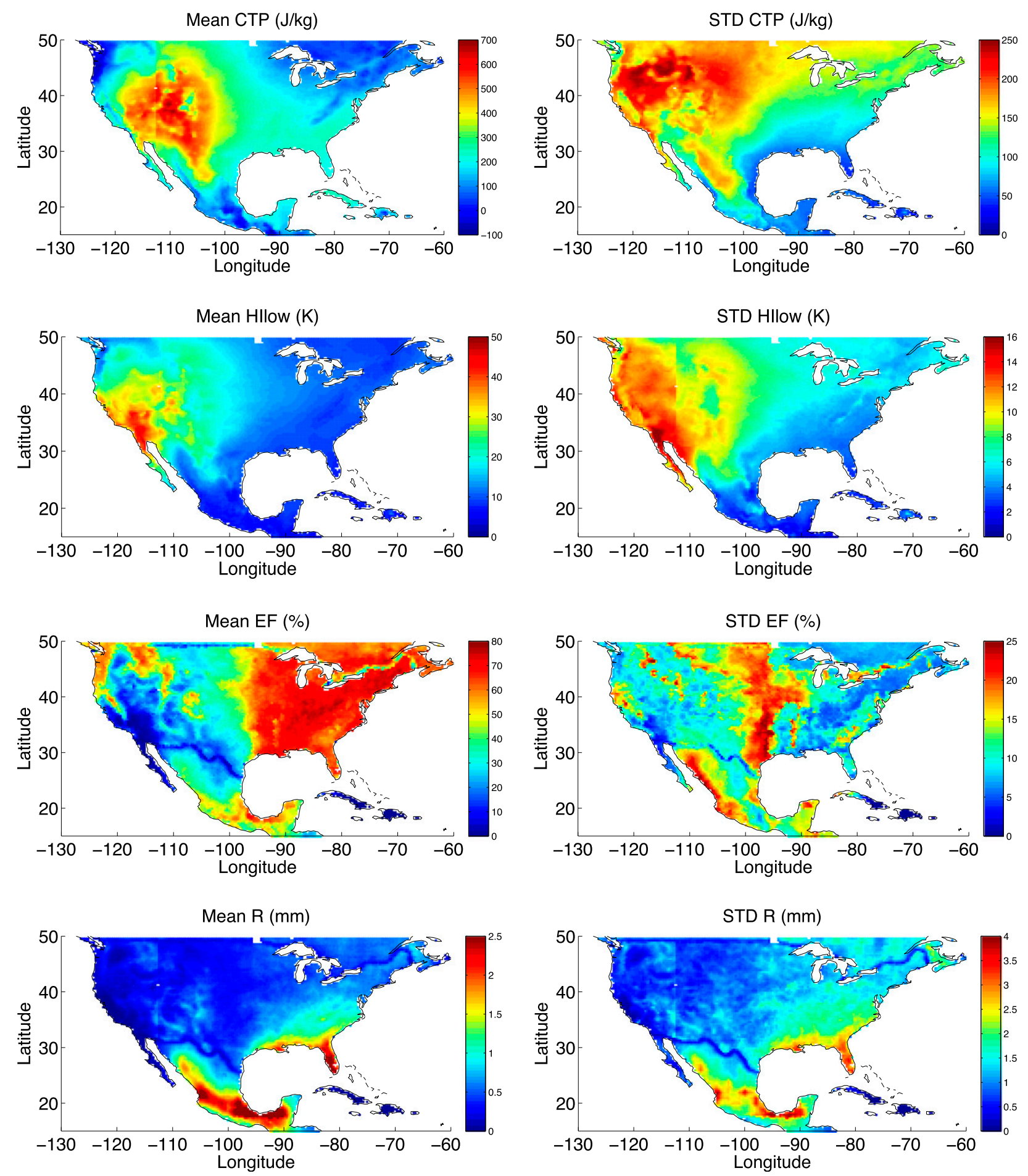

FIG. 1. (top to bottom) The CTP, HIlow, EF, and $R$ (left) monthly average and (right) standard deviation for June 1979. The quantity EF is defined as $\lambda \mathrm{E} /(H+\lambda \mathrm{E})$, where $\lambda \mathrm{E}=$ evaporative heat flux and $H=$ sensible heat flux.

points with negative CTP are excluded because they are too stable to support locally driven convection, indicating that any afternoon precipitation on these days is likely related to synoptic-scale systems and not to local conditions.
In addition to the complete CONUS domain (referred to hereafter as the Total domain), two regional domains are also considered: Florida $=25^{\circ}-31^{\circ} \mathrm{N}$ and $84^{\circ}-80^{\circ} \mathrm{W}$ and Missouri $=36^{\circ}-40^{\circ} \mathrm{N}$ and $94^{\circ}-90^{\circ} \mathrm{W}$. These two regions 
TABLE 1. Correlation between predictors (CTP, HIlow, and EF) and rainfall $R$.

\begin{tabular}{lccr}
\hline & HIlow & EF & \multicolumn{1}{c}{$R$} \\
\hline & & Total & \\
CTP & $0.6739 \pm 0.0003$ & $-0.4120 \pm 0.0005$ & $-0.1360 \pm 0.0005$ \\
HIlow & & $-0.4665 \pm 0.0004$ & $-0.2289 \pm 0.0005$ \\
EF & & & $0.1711 \pm 0.0005$ \\
& & Florida & \\
CTP & $0.5547 \pm 0.0036$ & $-0.0982 \pm 0.0052$ & $-0.0878 \pm 0.0052$ \\
HIlow & & $-0.2217 \pm 0.0050$ & $-0.1714 \pm 0.0051$ \\
EF & & & $0.1365 \pm 0.0051$ \\
& & Missouri & \\
CTP & $0.3008 \pm 0.0039$ & $-0.0996 \pm 0.0043$ & $0.0116 \pm 0.0043$ \\
HIlow & & $-0.1251 \pm 0.0043$ & $-0.1466 \pm 0.0043$ \\
EF & & & $0.0507 \pm 0.0043$ \\
\hline
\end{tabular}

are very different in their climatological behavior and demonstrate the limitations of the global analysis applied to the total domain. While Florida has its peak rainfall during the late afternoon hours (4-6 p.m.), much of the Midwestern CONUS has a nocturnal maximum of rainfall (see, e.g., Fig. 3 in Ruane 2010; see also Lee et al. 2008; Dai et al. 1999; Jiang et al. 2006). Factors contributing to this nocturnal maximum include the Rocky Mountains acting as an elevated heat source, absorbing radiation and initiating ascent over the mountains, and large-scale descent downwind over the Great Plains during the afternoon (Ruane 2010). Convective systems generated over the Rockies subsequently propagate eastward, allowing CAPE built up during the day to be released during the night (Dai et al. 1999; Jiang et al. 2006). Our global analysis considers only three input variables (EF, CTP, and HIlow) and as such does not explicitly account for dynamical suppression of locally driven afternoon convection over the Great Plains via forced large-scale descent. However, this suppression may in fact diminish the relationship between surface fluxes and afternoon rainfall that is captured in much of the rest of the domain, most notably Florida.

\section{d. Simple linear correlation analysis}

Table 1 summarizes the correlation among predictors (CTP, HIlow, and EF) and rainfall $R$ for the three spatial domains: Total, Florida, and Missouri. In the Total domain, CTP and HIlow are highly correlated to each other (0.67) as low-level moisture impacts the convective instability, EF appears to be slightly less dependent on CTP and HIlow ( -0.41 and -0.47$)$. None of the three input variables is strongly correlated to $R$ (maximum is -0.23 for HIlow and $R$ ). As a consequence, the relevance of these variables to the retrieval of rainfall information is limited. The correlations in the Florida domain are similar in pattern but their magnitudes are lower. For the Missouri domain, the correlations are even lower (e.g., 0.3 for CTP and HIlow instead of 0.67); this means that the system described by CTP, HIlow, and $\mathrm{EF}$ is less constrained and that other factors are acting in this region. The information provided by CTP, HIlow, and EF is not sufficient to characterize directly the rainfall $R$ in any of the regions.

First attempts to retrieve rainfall from the (CTP, HIlow, EF) combined information have been made using a metamodel. The results (not shown) are poor: the percentage of variance of $R$ that is explained by either a linear regression or neural network is equal to $10 \%$ and $13 \%$, respectively. The standard deviation of neural network retrieval errors for $R$ is about $1.28 \mathrm{~mm}$, almost as large at the natural variability of $R$, equal to $1.37 \mathrm{~mm}$. The difficulty to relate this raw data (CTP, HIlow, EF) to $R$ is not a surprise and illustrates well the need to perform the sensitivity analysis on statistical properties of rainfall (i.e., average intensity and frequency) instead of rainfall itself. This is the objective of the following section.

\section{e. Binning and rainfall intensity and frequency statistics}

In this section, some tests are performed to capture the statistical nature of the (CTP, HIlow, EF) to $R$ relationship. The goal of SA will be to reproduce this relationship. The variables CTP, HIlow, and EF are first binned. CTP variability range is divided into six bins: $0 \leq$ $\mathrm{CTP}<100 ; 100 \leq \mathrm{CTP}<200 ; 200 \leq \mathrm{CTP}<300 ; 300 \leq$ $\mathrm{CTP}<400 ; 400 \leq \mathrm{CTP}<500$; and $500 \leq \mathrm{CTP}$. HIlow is divided into seven bins: HIlow $<5 ; 5 \leq$ HIlow $<10$; $10 \leq$ HIlow $<15 ; 15 \leq$ HIlow $<30 ; 30 \leq$ HIlow $<40$; $40 \leq$ HIlow $<50$; and $50 \leq$ HIlow. The binning of EF is performed using 10 bins of $10 \%$ from $0 \%$ to $100 \%$.

The expected (mean) rainfall intensity, $E(R)$, and probability, $P(R)$, are estimated for each of the aforementioned bins. A point is considered to be rainy when $R>1 \mathrm{~mm}$. The expected rain intensity $E(R)$ is computed only for data points with rain, so the notation should be [E(R), R $\geq 1 \mathrm{~mm}]$ but $E(R)$ is used here for clarity of presentation. By computing these statistics, $E(R)$ and $P(R)$, for each bin, a large portion of the CTP, HIlow, and $E F$ variability is suppressed. The intrabin variability is not assessed, as only the interbin variability is analyzed in the following. The former can be the result of many factors that are not included in the analysis; for example, not all the geophysical variables involved in the processes have been accounted for or some spatiotemporal integration mechanisms are not considered.

Figure 2 depicts the expectation and probability statistics for the Total domain. The general trend for rainfall intensity $E(R)$ (upper row) is positive as EF 
$E(R)(m m)$ for $E F=5 \%$
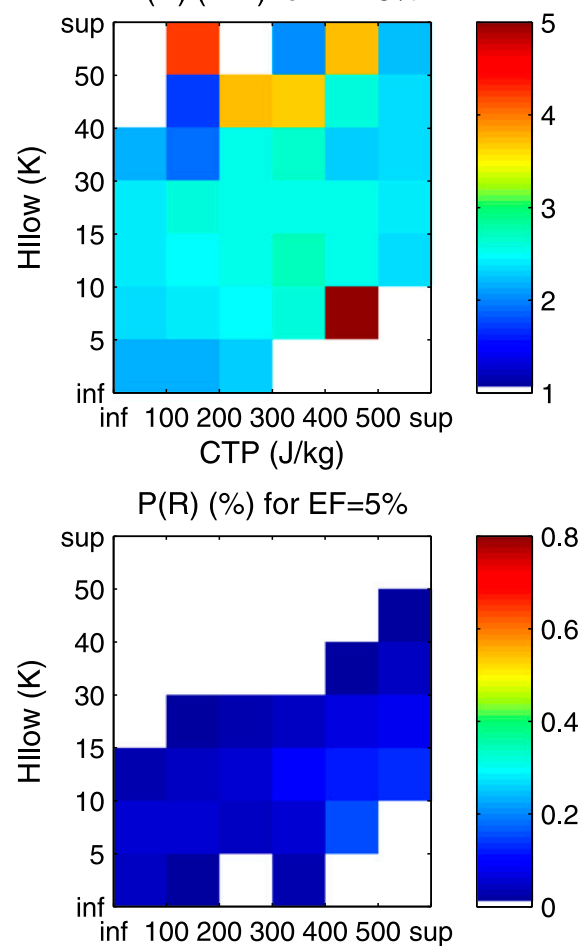

$E(R)(m m)$ for $E F=45 \%$

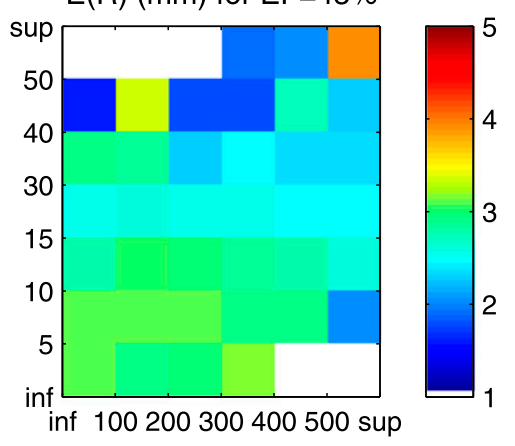

$P(R)(\%)$ for $E F=45 \%$

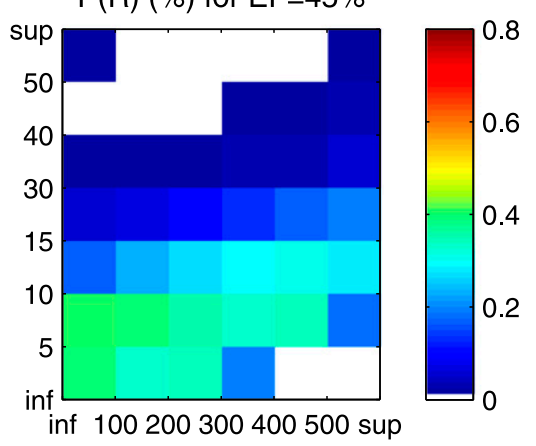

$E(R)(m m)$ for $E F=75 \%$

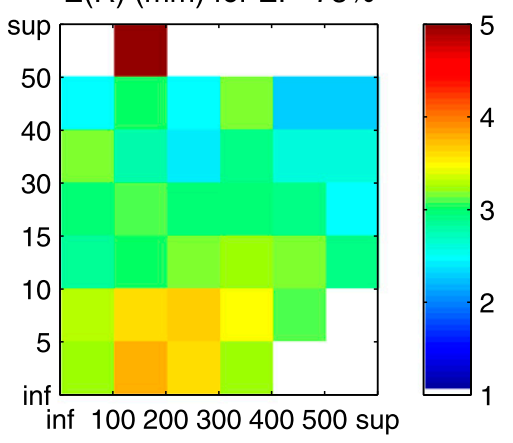

$P(R)(\%)$ for $E F=75 \%$

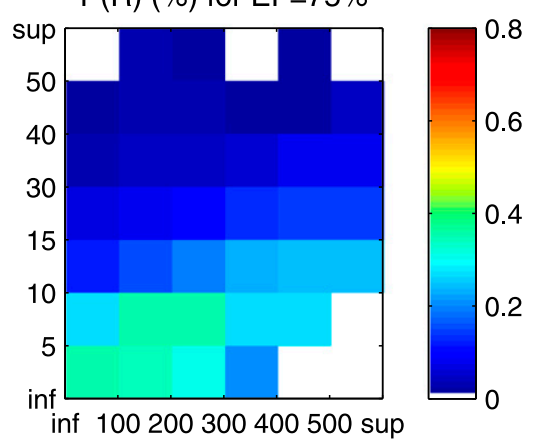

FIG. 2. (top) Total domain expected rainfall $E(R)(\mathrm{mm})$ with respect to binned CTP and HIlow for (left to right) $\mathrm{EF}=$ low $(5 \%)$, medium (45\%), and high (75\%). (bottom) As in top, but for rainfall probability $P(R)$ (values between 0 and 1 ).

increases. The increase of $E(R)$ with $\mathrm{EF}$ can be as large as $1 \mathrm{~mm}$, or up to a $30 \%$ increase in expected rainfall intensity. It should be noted that the pattern is noisy, especially in the limits of the binning domain, and the estimation in some bins can suffer from limited sampling in the bin. However, the general positive trend with EF is robust. The impact of EF is maximal for low HIlow and CTP levels. For the frequency (lower row of Fig. 2) the general pattern is less noisy. There is again a positive trend, with $P(R)$ increasing when EF goes from low to medium values. Only low values of HIlow are impacted, especially for low CTP.

Figures 3 and 4 are analogous to Fig. 2 but for the Florida and Missouri domains. Here the binning range is smaller given the lower range of variability inherent in the variables on more limited spatial scales. The behavior in the Florida domain is similar to the total domain but with stronger signatures in both $E(R)$ and $P(R)$. Note that $E(R)$ can go from 1 to $5 \mathrm{~mm}$ due to a change in EF and $P(R)$ can be increased by $20 \%$ when EF increases by $10 \%$. These results are compatible with Findell et al.'s (2011) results. On the other hand, in the Missouri domain, the behavior is different. The rainfall intensity $E(R)$ has a small increase from low to medium EF but the pattern is very noisy and the amplitude of changes is limited. Moreover, $P(R)$ does not seem to be related to CTP, HIlow, or EF. This is consistent with the information provided earlier about large-scale afternoon descent in this region largely suppressing locally driven convection.

Table 2 presents the correlations and their uncertainties for the binned predictors (CTP, HIlow, and $\mathrm{EF})$ and the rainfall expected intensity $E(R)$ and frequency $P(R)$ for the Total, Florida, and Missouri domains. As the structure of correlations in this table is very similar for the Total and the Florida domains, let us first consider these two regions. Among the three predictors, HIlow provides the best information on rainfall intensity $(-0.57$ and -0.52$)$. Over the ocean, it is well known that lower-level (ABL) humidity is a good predictor of convective triggering (Muller et al. 2009) but overall, to our knowledge, the link has not been as clear. For the rainfall frequency, again, HIlow is the more informative: -0.67 and -0.72 . These correlations are negative, so a decrease of HIlow (the low-level humidity increases) tends to increase the intensity and frequency of rainfall. EF has a significant correlation $(0.32$ and $0.35)$ for rainfall frequency only, not intensity. The correlation is positive, which means that when EF increases (i.e., wetter soils and more evaporation) the 

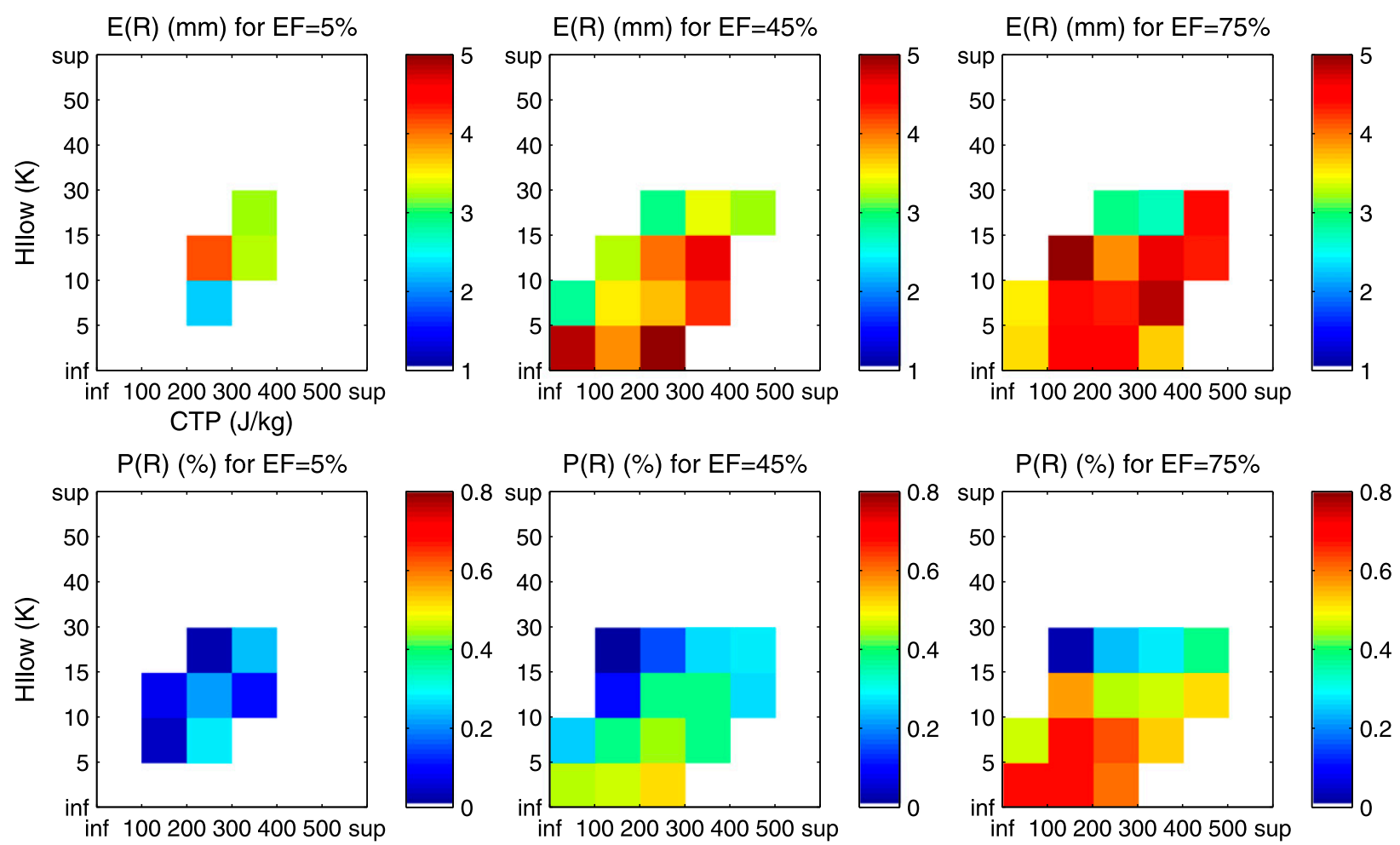

FIG. 3. As in Fig. 2, but for the Florida domain.

afternoon rainfall frequency increases but there is little impact on rainfall intensity. When considered CTP alone does not seem to provide any information on rainfall. This table is consistent with the averaged maps of Fig. 1.

For the Missouri domain, the correlation behavior is distinct from either the Total or Florida values: $E(R)$ is highly correlated to EF (0.75), to CTP (0.69), and to HIlow (0.38). Note that the correlation of $E(R)$ with HIlow is here positive, in contrast to the Total and Florida domains. For the rainfall frequency $P(R)$, the correlations are rather different too: 0.62 with CTP and $\mathrm{EF}$ and no correlation with EF. The strong connection between CTP and rainfall in this domain may stem from a relationship between large-scale atmospheric features and the CTP's characterization of low-level earlymorning atmospheric stability. This variable was not designed to assess large-scale vertical motion descent, but the positive correlations seen between CTP and rainfall features in the Missouri region may indicate that the two are in fact correlated.

It is important to note that even for the stronger of these correlations [e.g., HIlow and $P(R)$ correlated at -0.72 in Florida] the relationship is not very informative: the percentage of variability of $P(R)$ that can be explained by HIlow alone is just $51 \%$. So the forecast of $E(R)$ or $P(R)$ is a true challenge. However, it will be seen in the following that it is possible to obtain good forecast of $E(R)$ and $P(R)$, first, by using the interactions between (CTP, HIlow, EF) and, second, by using nonlinear models instead of linear correlations.

It is clear from the analysis of this section that the triggering of convection is not the same for the three spatial domains considered in this paper. This confirms the results in Figs. 2, 3, and 4. These differences in behavior mean that some other parameters are important to describe rainfall. It is beyond the scope of this paper to investigate which factors may account for these differences, but we intend to pursue this in future work. The main goal of the next section will be to define generic tools to estimate the sensitivities of the system defined in the Total domain.

\section{Results of the neural network sensitivity analysis}

\section{a. Neural network metamodel over the complete} domain

In this section, the analysis focus on the Total domain and on the relationships defined in Fig. 2. Linear and neural network $(\mathrm{NN})$ models are used to represent the relationships linking binned CTP, HIlow, and EF and 
$E(R)(m m)$ for $E F=5 \%$

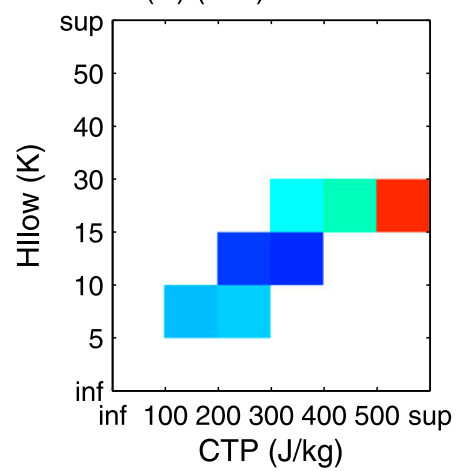

$P(R)(\%)$ for $E F=5 \%$

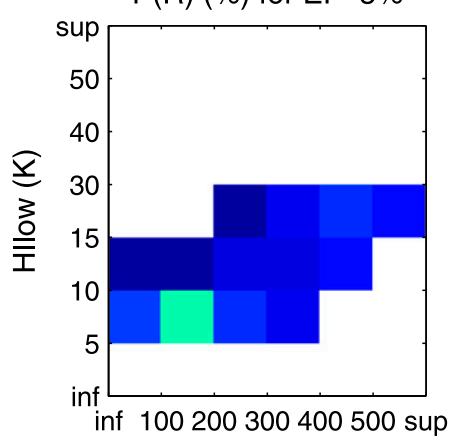

$E(R)(m m)$ for $E F=45 \%$

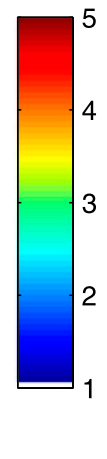

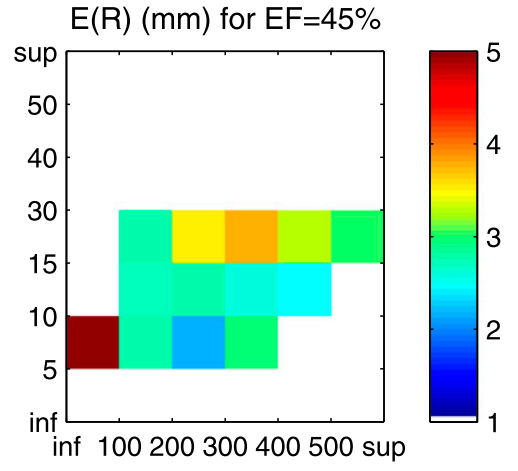
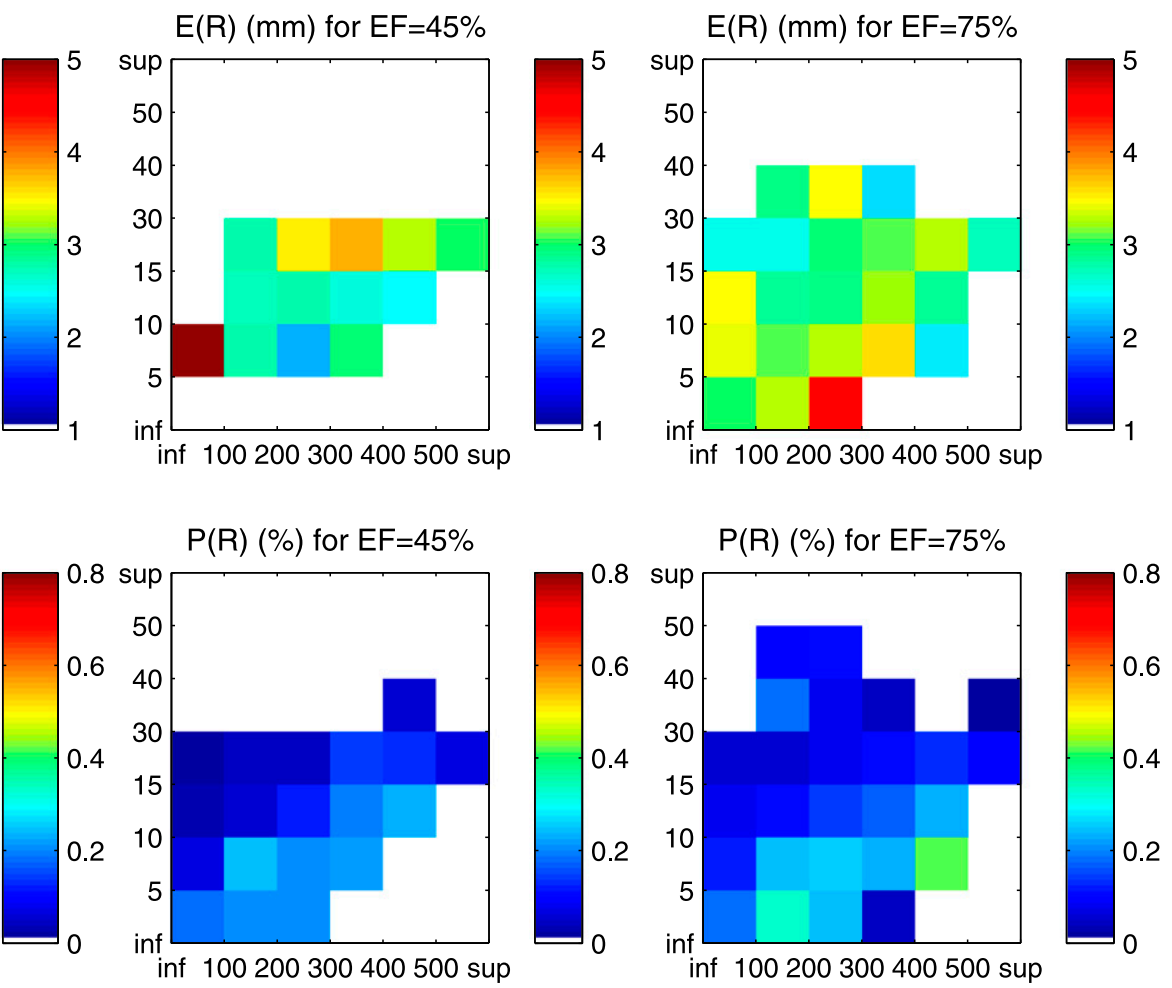

FIG. 4. As in Fig. 2, but for the Missouri domain.

afternoon rainfall, for both the expected intensity $E(R)$ and frequency $P(R)$. The models have three inputs and one output. A model is used for the retrieval of $E(R)$ and another for $P(R)$ (a simultaneous retrieval could also have been performed using a single model) so a total number of four models are tested. The two neural networks have been chosen with 10 neurons in the hidden layer (see appendix). This is a reasonable compromise providing a good approximation but not too many degrees of freedom in the metamodel. The training of the
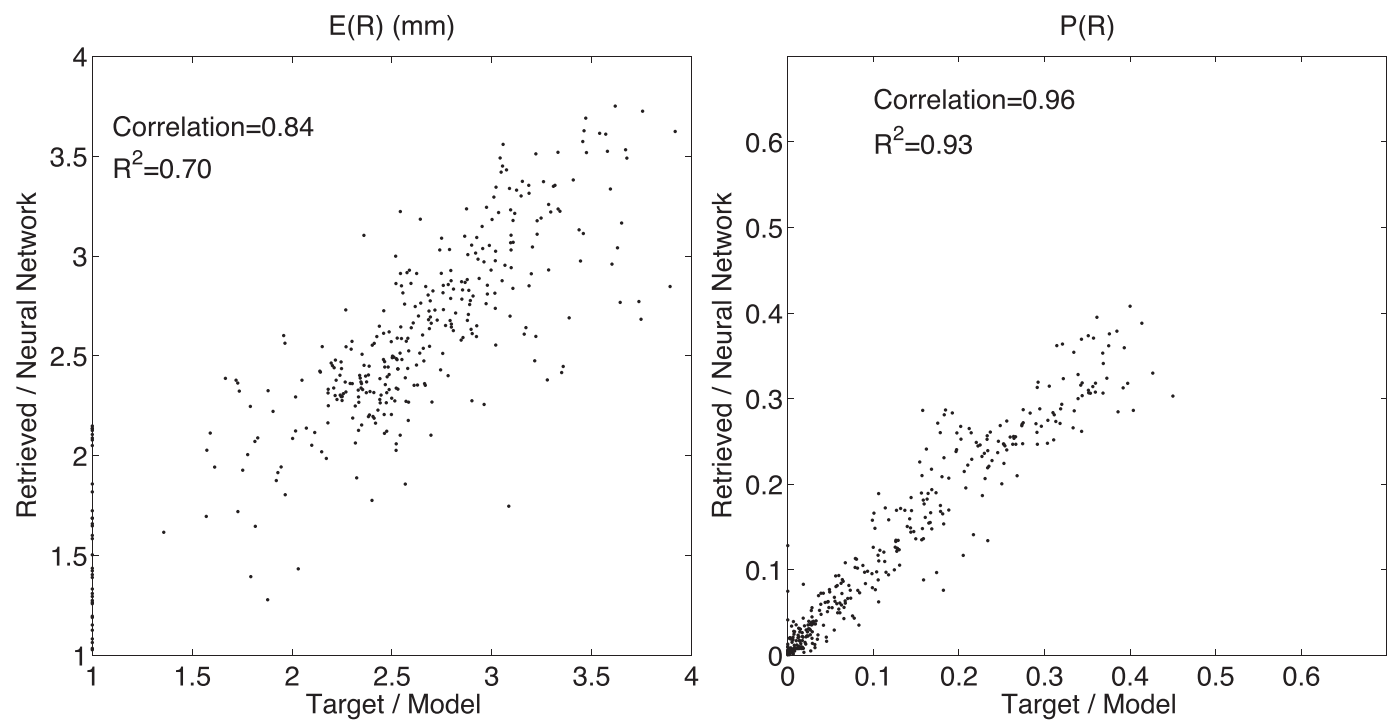

FIG. 5. Scatterplot of the NN retrieved vs target expected rainfall (left) intensity and (right) frequency. The correlation and the $R^{2}$ between retrievals and NARR targets are also indicated. 
TABLE 2. Correlation between predictors (CTP, HIlow, and EF) and rainfall intensity $E(R)$ and frequency $P(R)$. The $95 \%$ confidence interval is also on the right.

\begin{tabular}{lrr}
\hline & \multicolumn{1}{c}{$E(R)$} & \multicolumn{1}{c}{$P(R)$} \\
\hline & Total & \\
CTP & $-0.06 \pm 0.10$ & $-0.03 \pm 0.10$ \\
HIlow & $-0.57 \pm 0.07$ & $-0.67 \pm 0.06$ \\
EF & $0.17 \pm 0.10$ & $0.32 \pm 0.09$ \\
& Florida & \\
CTP & $0.03 \pm 0.18$ & $-0.04 \pm 0.18$ \\
HIlow & $-0.52 \pm 0.15$ & $-0.72 \pm 0.10$ \\
EF & $0.12 \pm 0.17$ & $0.35 \pm 0.15$ \\
& Missouri & \\
CTP & $0.69 \pm 0.07$ & $0.62 \pm 0.08$ \\
HIlow & $0.38 \pm 0.11$ & $0.07 \pm 0.13$ \\
EF & $0.75 \pm 0.05$ & $0.62 \pm 0.08$ \\
\hline
\end{tabular}

four models uses the binned dataset of section $3 \mathrm{e}$ because $E(R)$ and $P(R)$ need to be estimated from binned data.

Before defining and analyzing the full models, an information content analysis of the inputs with respect to $E(R)$ and $P(R)$ can be performed using the metamodels. The percentage of variance explained $\left(R^{2}\right)$ statistics of the linear and neural network models are listed in Table 3. This method can be used to define which input variables are most related to rainfall by quantifying the strength of each link, and measuring the information synergy among the variables. An advantage of this approach is that there is no need for using normalization coefficients in order to measure the significance of the variables. A disadvantage is that if two input variables are correlated, their combination may not improve the results even if a causal link exists with the output. Not surprisingly, the NN models outperform the linear models. However, the linear and NN $R^{2}$ are similar in magnitude for individual inputs, except for the retrieval of $P(R)$ using HIlow. Thus, nonlinearity of the $\mathrm{NN}$ is important not because of the nonlinear shape of the NN but because of the interaction terms. For instance, the retrieval of $P(R)$ using (CTP, HIlow, EF) provides a $R^{2}=0.71$ for the linear model and 0.93 for the NN model; $P(R)$ is better retrieved than $E(R)\left(R^{2}=93 \%\right.$ compared to $70 \%$ ). The strong agreement between the data and $\mathrm{NN}$ forecast model indicates that rainfall frequency is strongly related to the chosen predictors (CTP, HIlow, and EF) when considering the Total domain, and that the binning of the predictors and the use of the rainfall statistics instead of the raw rainfall is very efficient (section $3 \mathrm{e}$ ). The $R^{2}$ statistics of Table 3 also confirm the correlations of Table 2: for the characterization of $E(R)$ and $P(R)$, HIlow is most important, followed by EF and then CTP.
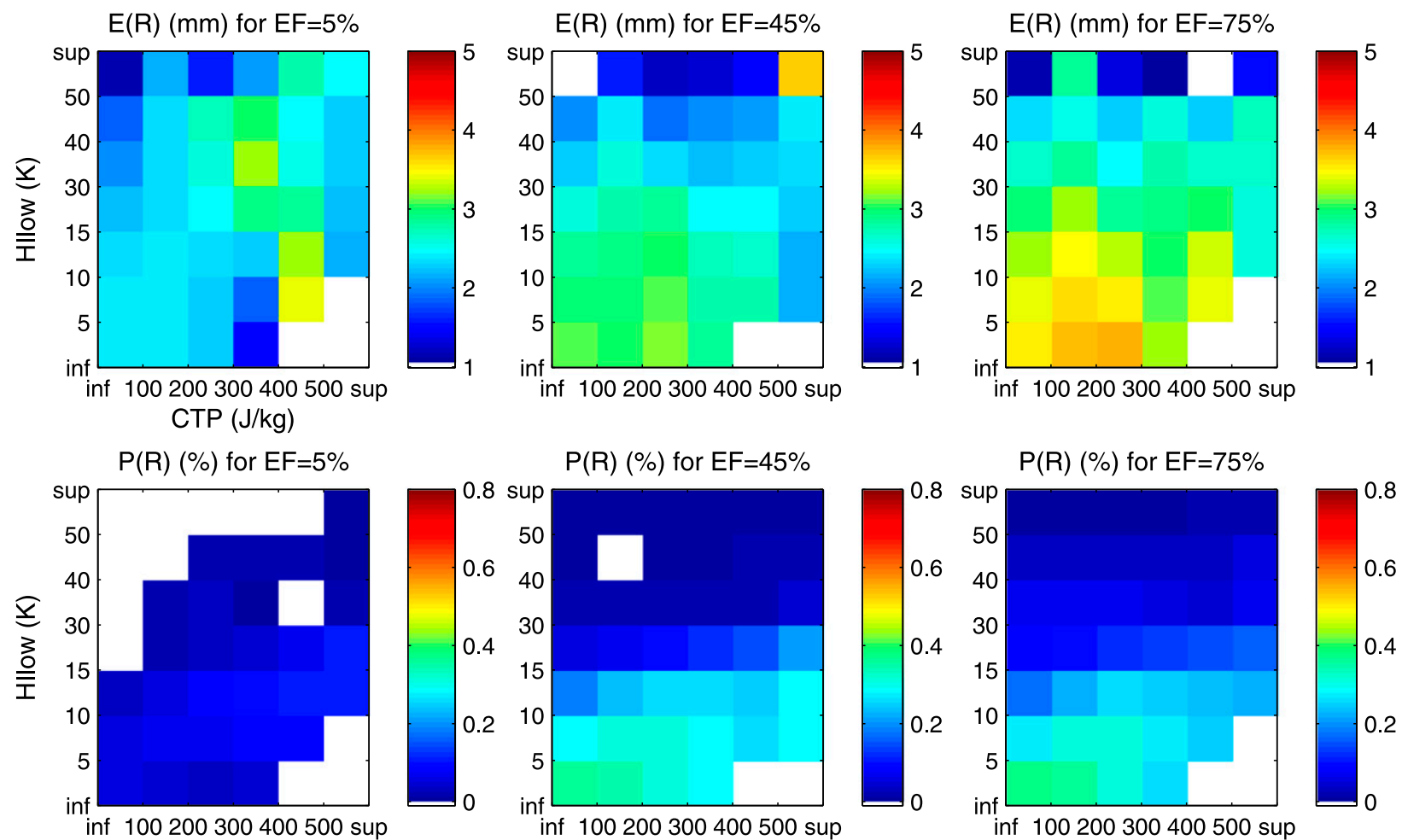

FIG. 6. As in Fig. 2, but for (top) retrieved expected rainfall $E(R)$ and retrieved rainfall probability. This figure is to be compared to real data in Fig. 2. 
TABLE 3. Values of $R^{2}$, the percentage of variance explained by the metamodel forecast [linear regression (LIN) or neural network $(\mathrm{NN})$ ], for the Total domain for the retrieval of rainfall intensity $E(R)$ and probability $P(R)$ when different combination of inputs are used.

\begin{tabular}{llllll}
\hline \hline \multirow{2}{*}{\multicolumn{1}{c}{ Inputs }} & \multicolumn{2}{c}{$E(R)$} & & \multicolumn{2}{c}{$P(R)$} \\
\cline { 2 - 3 } \cline { 6 - 7 } & LIN & NN & & LIN & NN \\
\hline CTP & 0.00 & 0.02 & & 0.00 & 0.00 \\
HIlow & 0.32 & 0.33 & & 0.45 & 0.70 \\
EF & 0.03 & 0.06 & & 0.10 & 0.14 \\
CTP + HIlow & 0.32 & 0.45 & & 0.45 & 0.75 \\
CTP + EF & 0.03 & 0.14 & & 0.10 & 0.15 \\
HIlow + EF & 0.34 & 0.52 & & 0.53 & 0.89 \\
CTP + HIlow + EF & 0.34 & 0.70 & & 0.71 & 0.93 \\
\hline
\end{tabular}

Figure 5 depicts the NN retrieval of the rainfall expected intensity (left) and frequency (right) against the NARR target. The correlations are also indicated: 0.84 $\left(R^{2}=70 \%\right)$ for expected intensity and $0.96\left(R^{2}=93 \%\right)$ for frequency of rainfall. The uncertainty for rainfall intensity is larger for lower values. The rainfall frequency model is in strong agreement with the data. This figure yields interesting physical insights onto the triggering and magnitude of rainfall. Based on those results, the frequency of rainfall can be predicted in NARR with a high degree of confidence if (CTP, HI, EF) are known, that is if we have information on the early morning stability, boundary layer humidity, and surface energy partitioning. On the other hand, the intensity of rainfall is much less confidently predicted with this triplet and additional information on the state of the atmosphere would be needed.

The NN forecast of the intensity and frequency statistics based on CTP, HIlow, and EF over the total domain is illustrated in Fig. 6. This figure can be directly compared to the original rainfall statistics in Fig. 2. It can be noted that the patterns for the rainfall intensity $E(R)$ are smoother than the original data, but they retain the same general behavior (i.e., increase of the expected intensity with increasing EF). This smoothing was to be expected: only 10 neurons are used in the hidden layer of the $\mathrm{NN}$ in order to limit the number of degrees of freedom in the model. This has a smoothing effect that is desirable in SA. The patterns of the rainfall frequency $P(R)$ are identical to the original patterns; they can barely be distinguished with the NARR original data (Fig. 2). This confirms that the NN metamodel $f$ reproduces very well the (CTP, HIlow, EF) to $P(R)$ relationship. The metamodel can therefore be used with confidence to estimate the sensitivities of the system.

It should be mentioned that experiments have been conducted to apply the Total domain metamodel of Fig. 6 on the Florida and the Missouri domains. As expected, the metamodel is not as good when applied to local domains (not shown) because the general behavior of Fig. 2 is not entirely consistent with the local behaviors of Figs. 3 and 4. As mentioned earlier, additional information appears to be necessary on the Florida and especially Missouri domains to characterize the differences of behavior described in sections $3 \mathrm{~d}$ and $3 \mathrm{e}$.

\section{b. Sensitivities of the general metamodel}

Since the NN metamodels are more accurate than the linear models, they are chosen here to estimate the sensitivities. Once the NN metamodels are trained to forecast rainfall expected intensity and frequency, it is simple to estimate the sensitivity of the outputs, $E(R)$ or $P(R)$, with respect to the three inputs, CTP, HIlow, and EF:

$$
\begin{aligned}
S_{E(R) / \mathrm{CTP}} & =\frac{\partial E(R)}{\partial \mathrm{CTP}}(\mathrm{CTP}, \text { HIlow }, \mathrm{EF}) \\
S_{E(R) / \mathrm{HIlow}} & =\frac{\partial E(R)}{\partial \text { HIlow }}(\mathrm{CTP}, \text { HIlow }, \mathrm{EF}) \\
S_{E(R) / \mathrm{EF}} & =\frac{\partial E(R)}{\partial \mathrm{EF}}(\mathrm{CTP}, \text { HIlow }, \mathrm{EF}) .
\end{aligned}
$$

Since the NN metamodels are relatively simple analytical functions, it is easy to obtain their analytical derivation. Similar formulas can be used to obtain rainfall probability $P(R)$ sensitivities instead of $E(R)$ in Eqs. (1). To better understand these sensitivities, normalizations are often used. This normalization can be quite complex, using input or output standard deviation, using global or pixel-level statistics, and the resulting normalized sensitivities can change significantly. Normalized sensitivities can be handy when comparing the relative strength of the sensitivities, but this easier comparison means that the sensitivities lose their physical unit and are highly dependent on the chosen normalization factor.

To represent the full coverage of these sensitivities, in particular the extreme cases, histograms of "sensitivity impact" have been estimated (Fig. 7). These sensitivity impacts are the raw sensitivities multiplied by a "characteristic" increment $\theta$ of its inputs: $\theta_{\text {CTP }} \times \partial P(R) / \partial \mathrm{CTP}$, for example. The characteristic increments have been chosen as $\theta_{\mathrm{CTP}}=+100 \mathrm{~J} \mathrm{~kg}^{-1}, \theta_{\mathrm{HIlow}}=+5 \mathrm{~K}$, and $\theta_{\mathrm{EF}}=$ $+10 \%$ (to avoid confusion, these characteristic increments are different than the binning steps). The statistics of Fig. 8 have been estimated for the full time record (1979-2003) and for the whole spatial domain.

It can be noted that a change of any of the three variables has a limited impact on $E(R)$ : only a fraction of precipitation results from these characteristic increases of CTP, HIlow, or EF. This is consistent with Figs. 2 and 6 where the dependency of $E(R)$ horizontally (i.e., for 

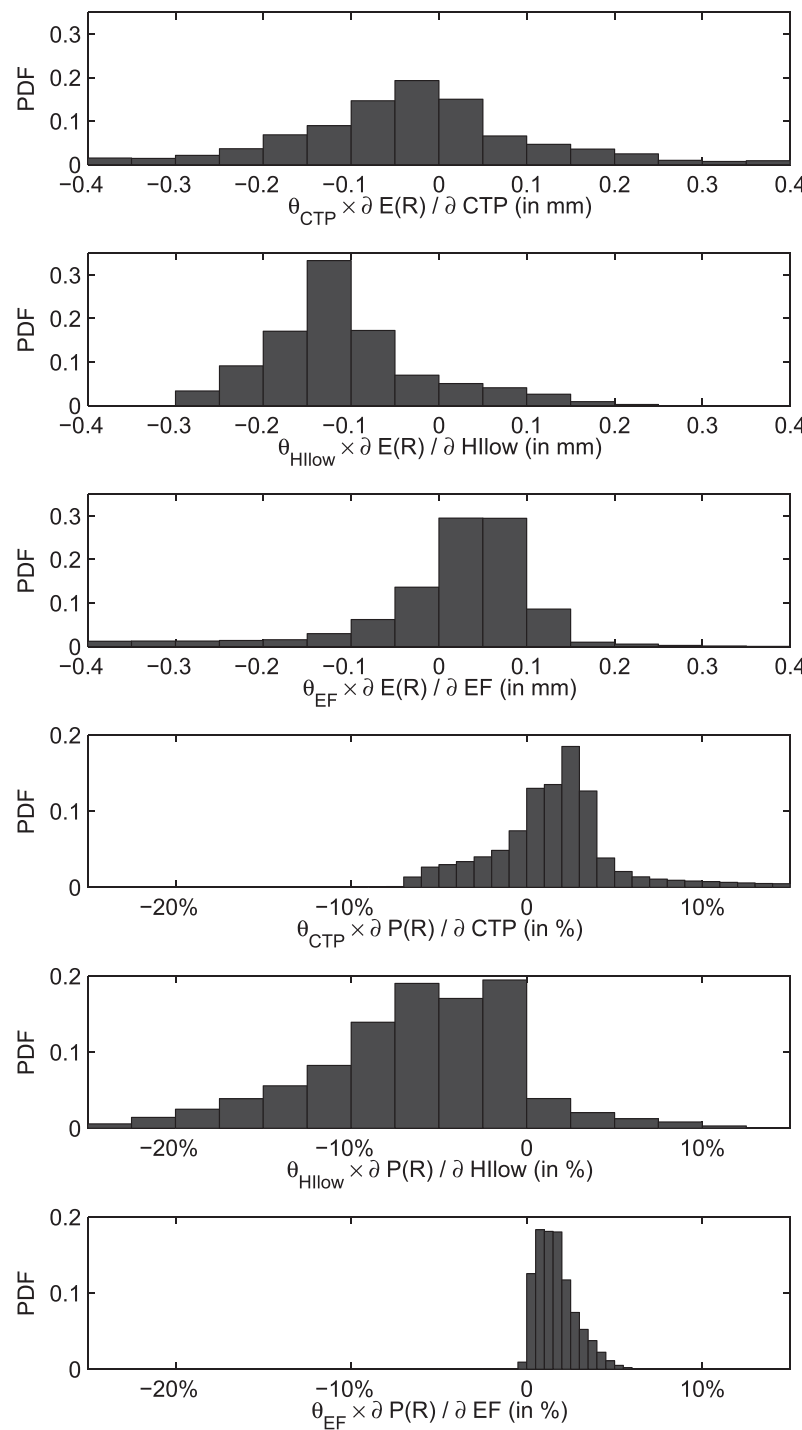

FIG. 7. Histogram of the "sensitivity impact" expressed as (top three panels) the expected rainfall $E(R)$ and (bottom three panels) rainfall frequency $P(R)$ sensitivities to (top to bottom) CTP, HIlow, and EF when multiplied by a characteristic input perturbation $\theta_{\text {СТP }}=+100 \mathrm{~J} \mathrm{~kg}^{-1}, \theta_{\text {HIlow }}=+5 \mathrm{~K}$, and $\theta_{\mathrm{EF}}=+10 \%$.

CTP) is very weak. The sensitivity of $E(R)$ to HIlow is most of the time negative, demonstrating that the behavior in the Missouri region is indeed anomalous. The dependency of $E(R)$ to $\mathrm{EF}$ is also weak, it is mainly positive but its impact is only significant for lower HIlow values (see Figs. 2 and 6). Significant increase of EF would be required to obtain a $0.5-\mathrm{mm}$ increase of expected rainfall $E(R)$.

The top three plots in Fig. 7 show the sensitivity impacts of $E(R)$ with respect to each of the three input variables (CTP, HIlow, and EF). The range of the $x$ axis $(-0.4$ to $0.4 \mathrm{~mm})$ indicates that each of the input variables has a limited impact on the expected amount of rainfall during a storm event. The histograms all span positive and negative values, indicating that the chosen characteristic increase in each of the three variables can lead to an increase or a decrease in $E(R)$, although the HIlow impacts are typically negative and the EF impacts are almost all positive. Despite the dominantly positive histogram, the small values indicate that very large EF changes would be required to produce just a $0.5-\mathrm{mm}$ increase of expected rainfall. This is consistent with the largely negligible values of the amplification feedback strength found in Findell et al. (2011).

It is important to note that the ranges of the sensitivities in Fig. 7 are dependent on the chosen increments $\theta_{\mathrm{CTP}}, \theta_{\mathrm{HIlow}}$, and $\theta_{\mathrm{EF}}$. In the right column of Fig. 1 , it can be seen that the standard deviation of variability in one pixel can be as large as $250 \mathrm{~J} \mathrm{~kg}^{-1}$ for CTP, $16 \mathrm{~K}$ for HIlow, and $25 \%$ for EF. This indicates that the characteristic perturbations used in Fig. 7 are quite reasonable and the sensitivity impact can be multiplied by 2 or 3 for extreme perturbations.

The sensitivity of $P(R)$ to CTP, HIlow, and EF appears more substantial (as shown earlier; see Fig. 5). The impact of CTP can be either positive or negative and it can have an impact of up to $10 \%$ on $P(R)$. HIlow impacts are almost always negative and can reduce $P(R)$ by more than $20 \%$. The sensitivity to EF is always positive and an increase of $10 \%$ of EF can lead to an increase of up to $5 \%$ in $P(R)$. This relationship between EF and $P(R)$ is consistent with the TFS results of Findell et al. (2011), although the magnitude of the impact is smaller with this characteristic EF perturbation than they saw when scaling their locally derived derivative with the local standard deviation of EF.

It is important to recognize that the sensitivities in Eq. (1) are partial derivatives. In general, increments in CTP, HIlow, and EF are linked and the sensitivities can add to or compensate each other. As a consequence, it is not possible to directly compare $\partial P(R) / \partial \mathrm{EF}$ (i.e., real partial derivative) and $\Delta P(R) / \Delta \mathrm{EF}$ (i.e., ratio of increments).

The spatial structures of these sensitivities are illustrated in Fig. 8. In each pixel, an averaged sensitivity has been estimated for the entire available period 19792003. This averaging has the tendency of limiting extreme cases so the ranges of variability in these maps are lower than for unscaled versions of the histograms of Fig. 7 but the spatial patterns obtained are relatively stable over time (not shown). The left column presents sensitivities on rainfall expected intensity, $E(R)$, and the right column for rainfall frequency, $P(R)$. The sensitivity of $E(R)$ and $P(R)$ to CTP, HIlow, and EF can vary significantly from one location to another. 

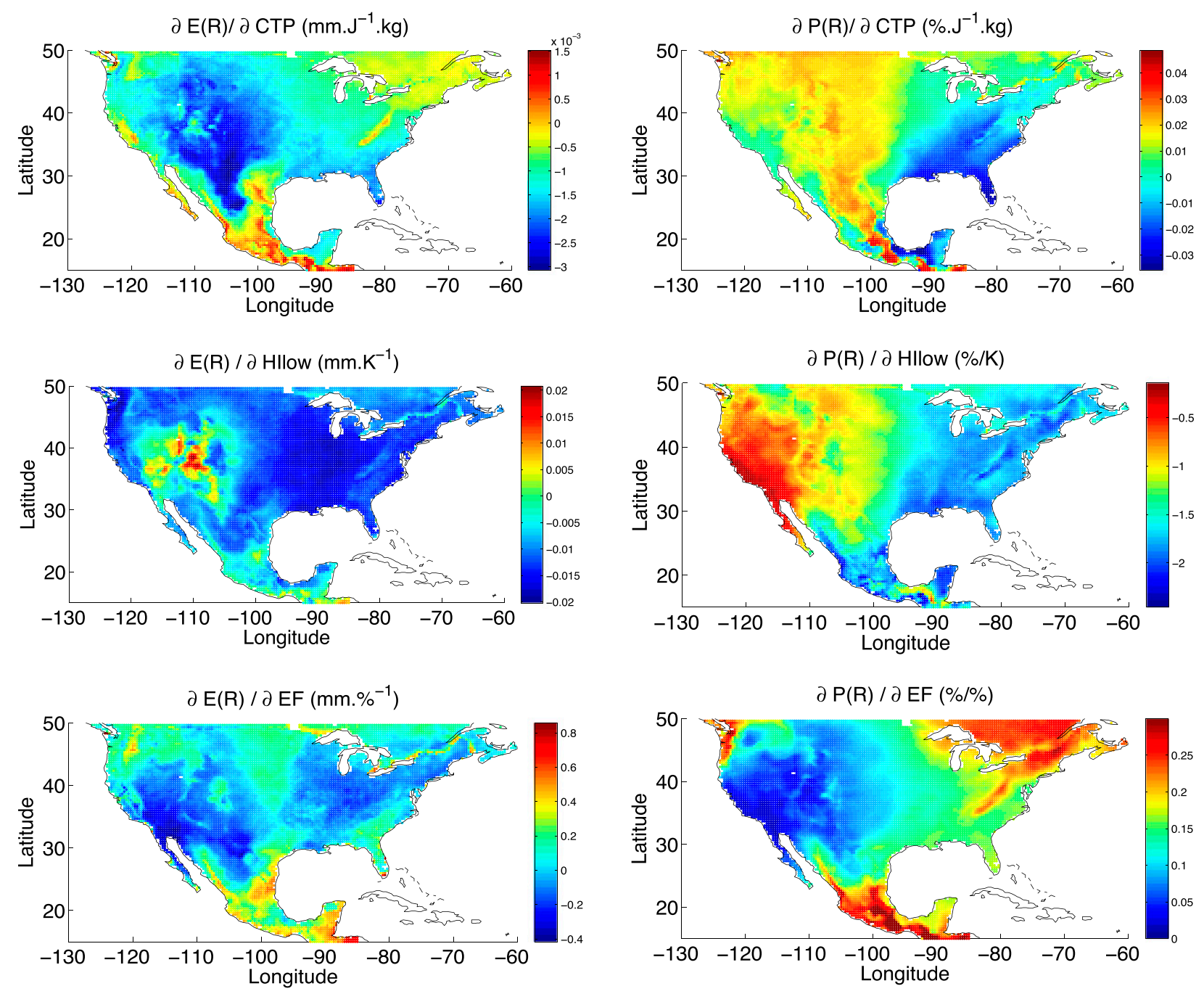

FIG. 8. Averaged rainfall (left) expected intensity $E(R)$, and (right) frequency $P(R)$ sensitivities for (top to bottom) CTP, HIlow, and EF [Eq. (1)]. The statistics were obtained using the full 1979-2003 dataset.

Let us first consider the rainfall intensity. Note that $E(R)$ was not correlated to CTP (Table 3) but the sensitivity appears to be significant in some of the regions. Atmospheric instability (as assessed by CTP) negatively affects the convective rainfall intensity over most of the domain, with the strongest impact in the Rocky Mountains and, to a lesser degree, Florida. The strongest linear correlation was with HIlow (-0.6); the sensitivities in Fig. 8 are consistent with that negative correlation, showing mostly negative sensitivities, especially in the East and Mexico. The sensitivity with EF is really bimodal, with a positive sensitivity in Mexico, the southeastern United States, the northwestern United States, and along a north-south band through the central United States, and a negative sensitivity in most of the western United States and in the region south of the Great Lakes. The feedback seen with soil moisture is going to be a composite effect of both the sensitivities to $\mathrm{EF}$, as well as the soil moisture-EF relationship.

The average sensitivity maps for $P(R)$ are represented in the right column of Fig. 8. Again, the correlation between $P(R)$ and CTP was negligible (Table 3 ) but the sensitivity is largely positive, in particular in the western half of the domain. This means that an increase in CTP typically increase rainfall frequency, with a bigger impact on Mexico and the western United States. This is consistent with the very definition of the CTP as an indicator of the convective instability. Indeed if the source of convection (plumes) originates the near surface, increasing instability will increase the likelihood of precipitation triggering. The effect is clearly nonlinear since it is expected that precipitation triggering in dryer atmosphere (West Coast) is less sensitive to an increase in atmospheric instability since in general the atmosphere is relatively 


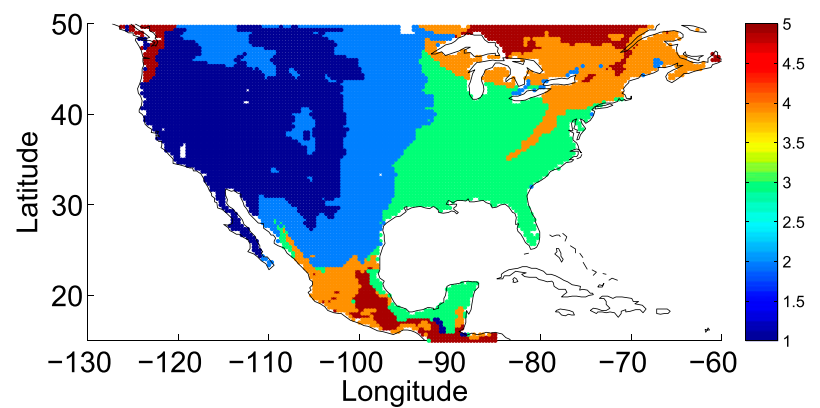

FIG. 9. Maps of land-atmosphere regimes based on the whole 1979-2003 series when five classes are used. From dark blue to dark red:1) atmospherically controlled,2) atmospheric + low-level humidity dependency, 3) low-level humidity sensitivity regime, 4) surface evaporative fraction + low-level humidity controlled, and 5) atmospheric + surface evaporative fraction dependency.

stable. The stronger linear correlation input with $P(R)$ was HIlow $(-0.67)$. This is consistent with the sensitivity map that includes only negative values, with very important magnitudes over the eastern United States and Mexico. A change in the lower tropospheric (boundary layer) humidity always leads to an increase in rainfall likelihood. This is consistent with recent findings of boundary layer control on the triggering of deep convection over the ocean (Peters and Neelin 2006; Muller et al. 2009).

The two maps at the bottom of Fig. 8 [sensitivities of $E(R)$ and $P(R)$ to EF] are similar to Fig. 1 in Findell et al. (2011) representing the sensitivity of convective triggering and rainfall depth to evaporative fraction. We obtain a similar general pattern for $P(R)$ but with much stronger positive feedback over the eastern United States and Mexico, and less over Florida. This highlights the differences discussed earlier raised by the grid cell approach of Findell et al. (2011) compared to the global approach used here. For $E(R)$, the sensitivities obtained in our paper seem to be less significant but again with a similar general spatial pattern.

\section{c. Sensitivity-based regimes of land-atmosphere interactions}

The situation dependency of the sensitivities makes the analysis more complex. To facilitate the interpretation of the land-atmosphere coupling, it is therefore useful to use a tool to synthesize results. To that end, we apply cluster analysis to sort the sensitivity data into regimes. These regimes are constructed to represent, as much as possible, the variability in the dataset. In this section, sensitivity-based regimes are obtained using the $K$-means algorithm on the sensitivities of $P(R)$ to CTP, HIlow, and $E F$. The rainfall frequency is preferred to the expected rainfall intensity because the metamodel is more reliable for $P(R)$ (section $4 \mathrm{a})$. The regimes are entirely defined by the three sensitivities (Fig. 8, right column); the absolute values of CTP, HIlow, EF, or $P(R)$ are not used to obtain them in the clustering process. The number of regimes, five, is chosen a priori.

The five regimes (Fig. 9) show a regional pattern reminiscent of the well-known map of dry versus wet soil advantage derived by Findell and Eltahir (2003a). While the choice of five clusters is subjective, an encouraging result is that these regimes are very stable when the number of clusters is increased: when adding regimes from one to five (not shown), the surface classification is described in better detail, with more transition areas, but the general structure remains stable, in particular with the eastern United States plus Mexico versus western United States contrast. This is a very good indicator of the robustness of the regimes. Furthermore, the clustering in Fig. 9 has been performed using averaged sensitivities (over 19792003) but when tested on daily values the spatial structures are again very stable.

The pattern of the five regimes in Fig. 9 closely resembles the pattern of EF sensitivity shown in the bottom right panel of Fig. 8. This indicates that the EF sensitivity is the dominant forcing determining the regimes. Additionally, the clustering algorithm orders the five regimes from weakest to strongest EF sensitivity (Table 4), again indicating the importance of this variable relative to the other two. Comparison of the relative importance of the three calculated sensitivities can be attempted in a variety of ways, each with its own strengths and weaknesses. The values of the regimemean sensitivities are provided in Table 4 both in terms of nonnormalized sensitivities and in terms of impact

TABLE 4. $P(R)$ sensitivity-based regimes.

\begin{tabular}{|c|c|c|c|c|c|c|c|}
\hline No. & Color & $\begin{array}{c}\frac{\partial P(R)}{\partial \mathrm{CTP}} \\
\left(\% \mathrm{~J}^{-1} \mathrm{~kg}\right)\end{array}$ & $\begin{array}{c}\frac{\partial P(R)}{\partial \text { HIlow }} \\
\left(\% \mathrm{~K}^{-1}\right)\end{array}$ & $\begin{array}{c}\frac{\partial P(R)}{\partial \mathrm{EF}} \\
\left(\% \%^{-1}\right)\end{array}$ & $\begin{array}{c}\theta_{\mathrm{CTP}} \frac{\partial P(R)}{\partial \mathrm{CTP}} \\
(\%)\end{array}$ & $\begin{array}{c}\theta_{\text {HIlow }} \frac{\partial P(R)}{\partial \text { HIlow }} \\
(\%)\end{array}$ & $\begin{array}{c}\theta_{\mathrm{EF}} \frac{\partial P(R)}{\partial \mathrm{EF}} \\
(\%)\end{array}$ \\
\hline 1 & Dark blue & 0.040 & -0.793 & 0.112 & 4.01 & -3.96 & 1.12 \\
\hline 2 & Light blue & 0.022 & -1.254 & 0.136 & 2.20 & -6.27 & 1.36 \\
\hline 3 & Green & -0.007 & -1.540 & 0.182 & -0.79 & -7.70 & 1.82 \\
\hline 4 & Orange & 0.013 & -1.616 & 0.245 & 1.37 & -8.08 & 2.45 \\
\hline 5 & Dark red & 0.031 & -1.313 & 0.271 & 3.11 & -6.56 & 2.71 \\
\hline
\end{tabular}


sensitivities (partial derivative multiplied by a characteristic increment $\theta_{\mathrm{CTP}}, \theta_{\mathrm{HIlow}}$, and $\left.\theta_{\mathrm{EF}}\right)$ as in Fig. 7. The global scaling approach of Table 4 should be interpreted with caution, keeping in mind the local information on the mean and variability of each variable provided in Fig. 1. For example, the characteristic increment of $5 \mathrm{~K}$ for $\theta_{\text {HIlow }}$ is about $1 / 3$ of the standard deviation of HIlow in the western part of the domain, but more than twice the standard deviation of HIlow in much of Mexico and the southeastern United States (Fig. 1d). A $\theta_{\mathrm{EF}}$ value of $10 \%$, on the other hand, is relatively close to the standard deviation of EF in all but the central swath of the domain. This means that the scaled columns of Table 4 will give greater weight to HIlow than to EF in Mexico and the southeastern United States. Additionally, given that the mean HIlow is relatively small in much of the domain (less than $10 \mathrm{~K}$ in Mexico and the entire eastern half of the domain) and is bounded by zero, distributions of HIlow for each grid cell are necessarily highly positively skewed, with long positive tails. The variability of highly skewed distributions is not appropriately captured by the standard deviation, which tends to be strongly impacted by these long tails. Thus, even Fig. 1d likely overstates the local characteristic variability of HIlow in much of the domain, and this further exacerbates the excess weighting of HIlow relative to EF in the scaled portion of Table 4 . Nevertheless, Table 4 provides useful insights into the characteristics of the five regimes determined from the clustering algorithm.

The two western regimes (dark blue and light blue) are both characterized by a weak positive dependence of $P(R)$ on EF, by a positive dependence of $P(R)$ on CTP that is stronger than in other domains, and by a negative dependence of $P(R)$ on HIlow that is weaker than that shown in other domains. The far western regime (dark blue) shows stronger dependence on CTP. In this region, rainfall is mostly atmospherically controlled while the transition regime running north-south through the center of CONUS (light blue) shows stronger dependence on HIlow. Convective rainfall is therefore determined by the stability and lower surface humidity. The three other regimes occupy Mexico and the eastern half of the domain. They all show negative relationships between $P(R)$ and HIlow (i.e., increased low-level humidity increases rainfall probability) and positive relationships between $P(R)$ and EF that are stronger than in the two western regimes. Given the difficulties discussed above regarding the different local variabilities and means of both EF and HIlow, assessing their relative importance is not straightforward; clearly both atmospheric humidity and surface flux partitioning are important players in determining the probability of rainfall in these three regimes. The largest difference between these three regimes is in the response to CTP: the green regime in the eastern United States has a very weak negative dependence between $P(R)$ and CTP, while the orange and dark red regimes have stronger positive $P(R)-\mathrm{CTP}$ relationships.

Comparing the regimes of Fig. 9 with the mean variable states shown in Fig. 1 is also instructive. The strong, positive $P(R)-\mathrm{CTP}$ relationships in the orange and dark red regimes are associated with mean CTP values near zero. The green regime, on the other hand, has mean CTP values around $200 \mathrm{~J} \mathrm{~kg}^{-1}$, but relatively small variability in CTP. One can also see the imprint of the mean HIlow and mean EF patterns on the regime map of Fig. 9: the regimes with relatively high mean EF also tend to have high sensitivity of $P(R)$ to variations in EF. This is consistent with the results of Findell et al. (2011).

While we have employed clustering to interpret differences in rainfall sensitivity in NARR, we note its potential applicability as a metric for model intercomparison and validation. The frequencies of occurrence of these regimes could be estimated in model outputs the potential differences could be measured. New regimes could also be estimated in model outputs and the two sets of regimes could be used compared.

\section{Summary and conclusions}

In this study, the relevance of three factors-evaporative fraction, convective triggering potential, and low-level humidity deficit- to afternoon convective rainfall intensity and frequency has been evaluated. It is shown that by binning these on three variables, it is possible to characterize the rainfall frequency and, to a lesser extent, the rainfall intensity. We have shown that it is better to perform the analysis using statistical properties of the rainfall (i.e., intensity expectation and frequency of occurrence) rather than on the raw rainfall values. This means that the process analyzed here is statistical in nature, which could aid in the development of new statistical (not deterministic) parameterizations in climate models (Palmer 2012).

Differences of behavior were found for the total (i.e., CONUS and Mexico) domain as well as the Florida and Missouri spatial domains. In particular, the sensitivity of convection to $\mathrm{EF}$ is high over Florida but equal to zero over Missouri. To fully capture the local behaviors within the framework of the full-domain analysis, additional information (e.g., vertical velocity) appears necessary.

The rainfall frequency can be highly controlled by the EF in some regions such as Florida and Mexico, confirming previous results from Findell et al. (2011), although the strength of the signal Findell et al. (2011) found in Florida was not matched in this analysis because the response of rainfall to EF described by the 
neural network is informed by data from all grid points in the domain. The very strong $d P / d$ EF sensitivity found in Findell et al. (2011) and Berg et al. (2013) is thus muted by the domain-wide analysis performed here.

Applying $K$-means clustering to the sensitivities of convective frequency and intensity to CTP, HIlow, and EF over the total domain, we found a gradient of landatmosphere coupling regimes over the continental United States. Five of these regimes were isolated and described; they are characterized by the convection sensitivity to lower-atmosphere stability, low-level humidity, and surface evaporative fraction. Furthermore, it was shown that a reliable statistical model based on neural networks can be used to represent the general relationships among these variables.

The sensitivity analysis (SA) approach used in this paper can be very valuable for climate studies: a simple statistical metamodel can be used to mimic the behavior of a complex climate system such as convection over land. It was shown that the nonlinearity of the model was essential in order to utilize interaction terms among the inputs. This metamodel can be used to 1) better understand the climate processes, 2) estimate the sensitivities of the system, and 3) develop process-oriented metrics to validate climate models against observations. These tools can facilitate model development because the emphasis is on the processes, not mean values of some variables. New models could become more stable, strengthening climate predictions.

The SA approach has multiple advantages:

- The analysis of the system is truly multivariate, meaning that the obtained sensitivities are true partial derivatives of the system (not, as is often the case in climate studies, increments of variables that would include the perturbation of all the system inputs).

- Because of the nonlinearity of the metamodel, the sensitivities that are obtained are state dependent (e.g., the obtained sensitivities are not the same over dry and wet environments).

- The SA can be used to quantify the importance for a process of many inputs; this allows identification of the most important factors in the climate system.

- SA can use observations or model outputs, which means that pertinent process-related metrics can be defined.

- Classical sensitivity estimation approaches used in climate studies handle well two variables problems, three at the best (Findell et al. 2011), but they could hardly be generalized to problems involving more variables. This would not be an issue for SA.

- The tools used in this study are conceptually very simple and easy to implement in practice. Furthermore, SA is much more generic and can be used in a straightforward way with other problems, whereas other approaches are dedicated to a very specific problem, such as in Findell et al. (2011).

Future directions for this work are numerous. First, other variables could be introduced in our analysis of the convection over CONUS. It is clear from the analysis that the combination of CTP, HIlow, and EF only is insufficient to fully characterize the intensity and triggering of convection. The tools developed in this paper can efficiently to determine the other important parameters controlling the convection over land. While there are potentially many factors controlling deep convection over land, as first steps, we intend to introduce some measures related to vertical velocity, the vertical structures of thermodynamic profiles, and boundary layer properties. The analysis could also use cloud fraction instead of rainfall.

It would be interesting to perform this analysis at a global scale, in order to study the prevalence of sensitivities identified for the North American domain or whether distinct sensitivities appear in different regions. Of course, to do so necessitates the use of datasets covering areas beyond North America.

The results obtained in this paper are based on the NARR reanalysis. It would be worthwhile to apply the methodology described here to additional datasets in order to assess how generalizable the finds may be. They could potentially differ for another model or pure observations and this methodology can be used to compare the sensitivities obtained for each one. This is very interesting to intercompare processes and facilitate model development.

An underlying motivation for this study was the development of physically based, process-oriented metrics of land-atmosphere coupling (and more generally, linkages in the climate system). The nonlinear, multivariate, state-dependent sensitivities estimated here may be useful for stimulating development of simplified metrics that can be readily applied across models or observations. Of course, it should be noted that the metamodel is fundamentally a statistical construct trained on a dataset. In fact, metamodels typically assess relationships as correlative rather than causal. For example, it is possible that the statistical link (or correlation) that is observed is related to hidden factors not considered in the analysis. To avoid such indirect correlations and obtain reliable causality links in the climate system, it may be possible to employ causality theory (Pearl 2009). This theory uses modern statistics and probability to define models (e.g., graph, causal, structural, and counterfactual models) and causation 
inference to analyze relationships between system constituent variables. Future work will evaluate the applicability of causal approaches to the climate system.

Acknowledgments. We thank Bill Rossow for interesting discussion about climate sensitivities, nonlinear systems, metrics, and feedback processes. We would like to than the three anonymous referees for their very important contribution in the improvement of this manuscript.

\section{APPENDIX}

\section{Method Overview}

\section{a. The concept of metrics}

In this paper, the term "metric" represents a tool that measures some generalized distance $d$ between a pair of datasets $D_{1}$ and $D_{2}, d\left(D_{1}, D_{2}\right)$. In addition to quantifying similarities or differences among datasets, metrics can be used to estimate a posteriori values of model parameters needed to bring a model in closer agreement with an observational target and to provide weightings for members in an ensemble of model runs. Simple metrics that facilitate understanding of the similarities and differences between two datasets are especially desirable. Much recent effort has been devoted to defining metrics for diagnosis of observed climate system processes and their evaluation and validation in models (Luo et al. 2012; van Herrwaarden et al. 2009).

Simple correlation can be used as a metric but the concept of metrics is more general. Correlation provides only the statistical link between two variables, and it is often a linear measure (although nonlinear correlation measures have been proposed). In this paper, the sensitivities that we derive are nonlinear (i.e., state dependent) and they involve simultaneously all the variables of interest.

\section{b. Sensitivity analysis}

While many approaches exist for analyzing relationships between two variables, $a$ and $b$, here we define sensitivity in terms of first partial derivatives $\partial b / \partial a$. Frequently in climate studies, the sensitivities $\partial b / \partial a$ are approximated using spatial and/or temporal increments of $a$ and $b$ : increments $\Delta a$ and $\Delta b$ are obtained from sampling two states of the climate system and the sensitivity is given by the ratio $\Delta a / \Delta b$.

Because the ratio $\Delta a / \Delta b$ is obtained by sampling two states of a model (or observations), the derived sensitivities are implicitly state dependent. Moreover, the incremental approach may be too limited because it relates $a$ and $b$, without any consideration of the other variables in the system. For example, the presence of feedbacks may complicate the interpretation of incremental sensitivities [see, e.g., van Herrwaarden et al. (2009), where the analysis of the land surface and convective clouds interaction has to take into account the land-atmosphere feedback with the influence of dryair entrainment]. An alternative approach, though one feasible only with models, involves directly perturbing the forcing applied to a model (e.g., changing top-of-theatmosphere solar radiation or imposing sea surface temperature perturbations). Synthetic experiments on soil moisture have been conducted by switching off the soil moisture dynamics (e.g., in GLACE) (Koster et al. 2004, 2006). The two states of the system permit estimation of $\Delta a$ and $\Delta b$ in order to obtain the sensitivity $\Delta a / \Delta b$. However, this approach may prove computationally expensive, especially in the context of model intercomparisons, and cannot be replicated with observations.

Ideally, sensitivity estimates should be robust to strong interactions between variables, nonlinearities including threshold behavior or saturation effects, and changes in background state or regime. To compare sensitivities from model outputs and observations, the method should be able to infer sensitivities from datasets from both model outputs and real observations. The SA applied here uses a reduced complexity metamodel that readily satisfies these criteria.

\section{c. Metamodel}

A metamodel $f$ is designed to represent the original system $S$ (Marrel et al. 2008, 2011). More specifically, $f$ is a function that approximates with some desired level of accuracy the outputs of $S$ but at substantially reduced computational cost (Kleijnen 2010; Simpson et al. 2001a,b; Storliea et al. 2009). Statistical models such as neural networks have been shown to be complex enough to represent complex climate processes; for example, $\mathrm{NNs}$ are able to predict ENSO with accuracies comparable to complex global climate models (Grieger and Latif 1994; Tang et al. 2000). From $f$, it is possible to estimate the sensitivities or uncertainties of $S$. Of greater significance from a process perspective, $f$ may elucidate the predominant control variables in $S$ as well as the optimal combination of inputs for predicting a particular output of $S$. On the other hand, because $f$ can detect noninfluential parameters, it may be used to simplify $S$. Metamodels are also called response surfaces, simplified models, emulators, proxy models, or surrogate models.

While $f$ can be determined mathematically by numerous techniques-for example, multiple linear regressions, polynomial approximations, splines, additive models, regression trees, support vector machines, Gaussian processes (Volkova et al. 2008), or neural 
networks (Bishop 1996) - the choice of $f$ is often dictated by application constraints. Because physical processes in climate may be nonlinear, the sensitivities of $S$ (or $f$ ) can be state dependent. For example, the sensitivity of convection over land to soil moisture is not the same for a dry or a wet atmosphere-soil (Findell and Eltahir 2003a,b). To represent such state-dependent sensitivities, it is necessary to implement a nonlinear model. Furthermore, the uncertainties present in the problem need to be taken into account. A distinction is often made between "local" and "global" SA (Saltelli et al. 2000): in the former, sensitivities are estimated for a single input point, while in the latter all input points are used together, under all conditions. In global analyses, the sensitivities are normalized by the standard deviation of the input and output variables and a socalled factor of importance is determined by the normalized sensitivity $F=\left(\sigma_{a} / \sigma_{b}\right)(\partial b / \partial a)$.

In an SA context, $f$ is often calibrated using model outputs but it can also be trained using a dataset of real observations. This allows any diagnostic based on $f$ to be used as a metric for comparing two full systems $S_{1}$ and $S_{2}$.

\section{d. Neural network analysis}

In this paper, a neural network (NN) statistical model is used to represent the multivariate and nonlinear relationships in the climate system: $b=\mathrm{NN}(a)$. This approach was first proposed in Aires and Rossow (2003) and has since been used, for example, in Chen et al. $(2003,2006)$. NN techniques have proved very successful in developing computationally efficient representation tools. The multilayered perceptron (MLP) model (Rumelhart et al. 1986) is selected here. It is a nonlinear mapping model: Given an input $a$, it provides a nonlinear output $b$. In this paper, an NN model with only one hidden layer will be considered (Fig. A1). Each layer in the $\mathrm{NN}$ is composed of individual neurons. A neuron performs first a weighted average of its inputs from the previous layer. The so-called synaptic weights are assigned to each connection between two neurons. These weights represent the NN parameters to be defined during the training stage. The NN chosen in this study is a fully connected MLP (i.e., every neuron has a connection with all neurons of the previous layer). Once this weighted average is performed, a nonlinear sigmoid function is applied. The final output of a neuron $i$ is given by

$$
b_{i}=\sigma\left(\sum_{j=1}^{N} w_{j, i} a_{j}\right) \text {, }
$$

where $a_{j} ; j=1, \ldots, N$ are the $N$ inputs of the neuron, $w_{j, i}$ is the synaptic weight between neuron $j$ and $i$, and $\sigma$ is

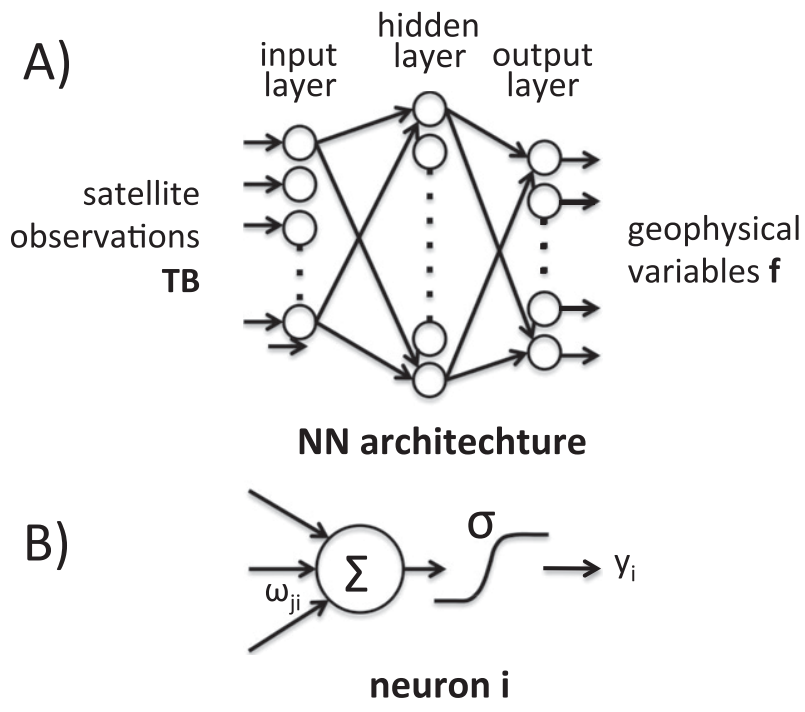

FIG. A1. Multilayered neural network perceptron (a) architecture and (b) neuron $i$.

a sigmoid function (Bishop 1996). Bias terms are also present in this model, but they are suppressed here for simplicity of presentation. The MLP model is defined by the number of input neurons (i.e., the size of the inputs, number of channels), the number of outputs (i.e., the size of the geophysical variables to retrieve), and the number of neurons in the hidden layers that control the complexity of the model. A study must be conducted to define the optimal number of neurons in the hidden layer. A balance needs to be found: too many free parameters in the model can result in overlearning (over parameterization), leading to degraded generalization properties. On the contrary, too few free parameters will yield under parameterization and bias error of the model.

The neural network used in this study can be represented by a very simple function: $b=f(a)=W_{2} \sigma\left(W_{1} a\right)$, where $W_{1}\left(W_{2}\right)$ is the matrix of weights from the input to the hidden layer (from the hidden to the output layer). The Jacobian of this function can be derived for any input state $a: \partial f(b) / \partial a=W_{2} \sigma^{\prime}\left(W_{1} a\right) W_{1}$, where $\sigma^{\prime}$ is the derivative of the sigmoid function $\sigma$. The state dependency of this Jacobian results from the presence of the sigmoid function $\sigma$, otherwise the model would be linear and the Jacobians would be constant for all states $a$. This state dependency is important, as the $\mathrm{NN}$ is able to adapt the sensitivity of its outputs based on the input state $a$. It can use only one source of information when the other sources are not pertinent or it can combine them in a nonlinear way when necessary. Complex interactions among the model inputs can be exploited by the NN.

The NN is trained to reproduce the behavior described by a database of samples composed of inputs and 
their associated outputs, $\left(a^{e}, b^{e}\right)$, for $e=1, \ldots, N$, with $N$ being the number of samples in the training database. Provided that enough samples (i.e., $N$ ) are available, any continuous relationship, as complex as it is, can be represented by an MLP (Hornik et al. 1989). Furthermore, a theorem of Cybenko (1989) shows that a two-hiddenlayer $\mathrm{NN}$ is able to represent any discontinuous function. A quality criterion that measures the discrepancies between the $\mathrm{NN}$ outputs and the desired targets from the learning dataset must be defined. In this paper, the weighted least squares criterion is used. This quality criterion is minimized during the learning of the NN. The learning algorithm used to train the $\mathrm{NN}$ is the classical "back propagation" algorithm. This optimization technique has long proved efficient for such applications (Bishop 1996).

\section{e. Local versus global analysis}

The distinction is often made between local and global sensitivity analysis (Saltelli et al. 2000): in local analysis, the sensitivities are estimated for a single input point. Global analyses intend to provide a sensitivity analysis at a global scale, for all types of situations, and taking into account their probabilities. For example, in global analyses, the sensitivities are normalized by the standard deviation of the input and output variables and the socalled factor of importance, which is given by the normalized sensitivity $F=\left(\sigma_{a} / \sigma_{b}\right)(\partial b / \partial a)$.

To test if the analysis performed in this paper, tests have been conducted at the CONUS, regional (i.e., Florida and Missouri), and pixel levels (not shown). There are no significant differences among the regional and pixel levels; this is to be expected since the two regions are hydrologically homogeneous. In this paper, the global sensitivities have been privileged because it allows determining functional relationships that are independent of the location and that describe the full range of behavior. However, the use of smaller regions (and contrasting these results with the global results) can help identify processes not included in the analysis but that should be included in future applications. For instance, the differences between Missouri and the global results indicate that variables/processes are missing that play an important role in this region, but not in the Florida region. We suggest that a vertical velocity-type term should be added to better characterize the Missouri region.

\section{f. Caveats}

Several caveats on the applicability of SA and the metamodel $f$ should be noted. First, the number of samples required may be very large. Thus, for sparsely sampled high-dimensional spaces optimal sampling strategies should be used (Aires and Prigent 2007; Paul and Aires 2013, manuscript submitted to Quart. J. Roy. Meteor. Soc.). In climate studies, especially those based on models, this problem is rarely an issue since large amounts of data are generally available. On the other hand, it is often not possible to represent very precisely the system $S$, as 1) some variables may not be available or may not be defined a priori (however, exploratory tools are available to investigate the more important factors in a physical relationship); and 2) relationships employed in $f$ may be too simplistic to represent complex physical mechanisms integrated spatially and temporally. However, the neural network models used in this study are universal approximation tools (Hornik et al. 1989, 1990; Cybenko 1989) so this is not an issue here.

By pooling a large dataset together and using statistics to find relationships among the variables, spurious correlations can appear:

- They can result from pure coincidental events in two variables; this is frequent when comparing not-longenough time series (in the time domain) or spatial patterns.

- This can be related to "indirect correlations": for example, a variable $V_{1}$ impacts $V_{2}$ that impacts itself $V_{3}$ (an indirect correlation between $V_{1}$ and $V_{3}$ can then be measured); or a variable $V_{1}$ impacts both $V_{2}$ and $V_{3}$ (an indirect correlation exist between $V_{2}$ and $V_{3}$ ).

By increasing the number of data in the dataset, pooling spatial and temporal samples together, the pure coincidental correlations are less probable. To avoid indirect correlations, other more sophisticated statistical approaches need to be used. We mention in the perspectives the "causality theory" that has been developed to handle such difficulties, avoid spurious correlations, and find relevant causal physical links among the variables. This technique will be tested in a forthcoming study.

\section{REFERENCES}

Aires, F., and W. Rossow, 2003: Inferring instantaneous, multivariate and nonlinear sensitivities for the analysis of feedback processes in a dynamical system: The Lorenz model case study. Quart. J. Roy. Meteor. Soc., 129, 239-275.

— spaces for satellite remote sensing database. J. Geophys. Res., 112, D20301, doi:10.1029/2007JD008391.

Berg, A., K. Findell, B. Lintner, P. Gentine, and C. Kerr, 2013: Precipitation sensitivity to surface heat fluxes over North America in reanalysis and model data. J. Hydrometeor., 14, 722-743.

Berg, L., and R. Stull, 2004: Parameterization of joint frequency distributions of potential temperature and water vapor mixing ratio in the daytime convective boundary layer. J. Atmos. Sci., 61, 813-828. 
Betts, A. K., and M. Silva Dias, 2010: Progress in understanding land-surface-atmosphere coupling from LBA research. J. Adv. Model. Earth Syst., 2 (6), doi:10.3894/JAMES.2010.2.6.

—_, J. Ball, A. Beljaars, M. Miller, and P. Viterbo, 1996: The land surface-atmosphere interaction: A review based on observational and global modeling perspectives. J. Geophys. Res., 101, 7209-7225.

Bishop, C., 1996: Neural Networks for Pattern Recognition. Clarendon Press, 482 pp.

Brubaker, K., and D. Entekhabi, 1996: Analysis of feedback mechanisms in land-atmosphere interaction. Water Resour. Res., 32, 1343-1357.

,$- \ldots$, and P. Eagleson, 1993: Estimation of continental precipitation recycling. J. Climate, 6, 1077-1089.

Chen, Y., J. Miller, J. Francis, G. Russell, and F. Aires, 2003: Observed and modeled relationships among Arctic climate variables. J. Geophys. Res., 108, 4799, doi:10.1029/2003JD003824.

_ , F. Aires, J. Francis, and J. Miller, 2006: Observed relationships between Arctic longwave cloud forcing and cloud parameters using a neural network. J. Climate, 19, 4087-4104.

Cook, B. I., G. B. Bonan, and S. Levis, 2006: Soil moisture feedbacks to precipitation in southern Africa. J. Climate, 19, 41984206.

Crago, R., 1996: Conservation and variability of the evaporative fraction during the daytime. J. Hydrol., 180, 173-194.

_ - and W. Brutsaert, 1996: Daytime evaporation and the selfpreservation of the evaporative fraction and the Bowen ratio. J. Hydrol., 178, 241-255.

Cybenko, G., 1989: Approximation by superpositions of a sigmoidal function. Math. Control Signals Syst., 2, 303-314.

Dai, A., F. Giorgi, and K. E. Trenberth, 1999: Observed and modelsimulated diurnal cycles of precipitation over the contiguous United States. J. Geophys. Res., 104 (D6), 6377-6402.

Dirmeyer, P. A., and K. L. Brubaker, 2007: Characterization of the global hydrologic cycle from a back-trajectory analysis of atmospheric water vapor. J. Hydrometeor., 8, 20-37.

—, R. D. Koster, and Z. Guo, 2006: Do global models properly represent the feedback between land and atmosphere? J. Hydrometeor., 7, 1177-1198.

- K. L. Brubaker, and T. DelSole, 2009: Import and export of atmospheric water vapor between nations. J. Hydrol., $\mathbf{3 6 5}$, $11-22$.

Dominguez, F., P. Kumar, X.-Z. Liang, and M. Ting, 2006: Impact of atmospheric moisture storage on precipitation recycling. J. Climate, 19, 1513-1530.

Ek, M., and A. Holtslag, 2004: Influence of soil moisture on boundary layer cloud development. J. Hydrometeor., 5, 86-99.

Eltahir, E., 1989: A feedback mechanism in annual rainfall, central Sudan. J. Hydrol., 110, 323-334.

— , and R. Bras, 1996: Precipitation recycling. Rev. Geophys., 34, 367-378.

Entekhabi, D., I. Rodriguez-Iturbe, and R. L. Bras, 1992: Variability in large-scale water balance with land surface-atmosphere interaction. J. Climate, 5, 798-813.

Ferguson, C., and E. Wood, 2011: Observed land-atmosphere coupling from satellite remote sensing and reanalysis. J. Hydrometeor., 12, 1221-1254.

Findell, K., and E. Eltahir, 2003a: Atmospheric controls on soil moisture-boundary layer interactions. Part I: Framework development. J. Hydrometeor., 4, 552-569.

_ and 2003b: Atmospheric controls on soil moistureboundary layer interactions. Part II: Feedbacks within the continental United States. J. Hydrometeor., 4, 570-583.
- P. Gentine, B. Lintner, and C. Kerr, 2011: Probability of afternoon precipitation in eastern United States and Mexico enhanced by high evaporation. Nat. Geosci., 4, 434-439, doi:10.1038/ngeo1174.

Gentine, P., D. Entekhabi, A. Chehbouni, G. Boulet, and B. Duchemin, 2007: Analysis of evaporative fraction diurnal behaviour. Agric. For. Meteor., 143, 13-29.

- _ — , and J. Polcher, 2010: Spectral behaviour of a coupled land-surface and boundary-layer system. Bound.-Layer Meteor., 134, 157-180.

,-- , and - 2011: The diurnal behavior of evaporative fraction in the soil-vegetation-atmospheric boundary layer continuum. J. Hydrometeor., 12, 1530-1546.

—, A. Betts, B. Lintner, K. Findell, C. van Heerwaarden, A. Tzella, and F. D'Andrea, 2013a: A probabilistic-bulk model of coupled convection. Part I: Clear-sky case. J. Atmos. Sci., 70, 1543-1556.

— A. A. M. Holtslag, F. D'Andrea, and M. Ek, 2013b: Surface and atmospheric controls on the onset of moist convection over land. J. Hydrometeor., 14, 1443-1462.

Grieger, B., and M. Latif, 1994: Reconstruction of the El Niño attractor with neural networks. Climate Dyn., 10, 267-276, doi:10.1007/BF00228027.

Guo, Z., and Coauthors, 2006: GLACE: The Global LandAtmosphere Coupling Experiment. Part II: Analysis. J. Hydrometeor., 7, 611-625.

— P. A. Dirmeyer, and T. DelSole, 2012: Rebound in atmospheric predictability and the role of the land surface. J. Climate, 25, 4744-4749.

Hohenegger, C., P. Brockhaus, C. S. Bretherton, and C. Schär, 2009: The soil moisture-precipitation feedback in simulations with explicit and parameterized convection. J. Climate, 22, 5003-5020.

Hornik, K., M. Stinchcombe, and H. White, 1989: Multilayer feedforward networks are universal approximators. Neural Networks, 2, 359-366.

,$- \ldots$, and -1990 : Universal approximation of an unknown mapping and its derivatives using multilayer feedforward networks. Neural Networks, 3, 551-560.

Jiang, X., N.-C. Lau, and S. A. Klein, 2006: Role of eastward propagating convection systems in the diurnal cycle and seasonal mean of summertime rainfall over the U.S. Great Plains. Geophys. Res. Lett., 33, L19809, doi:10.1029/2006GL027022.

Katul, G. G., R. Oren, S. Manzoni, C. Higgins, and M. B. Parlange, 2012: Evapotranspiration: A process driving mass transport and energy exchange in the soil-plant-atmosphere-climate system. Rev. Geophys., 50, RG3002, doi:10.1029/2011RG000366.

Kleijnen, J., 2010: Design and Analysis of Simulation Experiments. Springer, $232 \mathrm{pp}$.

Koster, R. D., and M. J. Suarez, 1994: The components of a 'SVAT' scheme and their effects on a GCM's hydrological cycle. $A d v$. Water Resour., 17, 61-78.

, and S. P. Mahanama, 2012: Land surface controls on hydroclimatic means and variability. J. Hydrometeor., 13, 1604-1620.

-, M. J. Suarez, R. W. Higgins, and H. M. van den Dool, 2003: Observational evidence that soil moisture variations affect precipitation. Geophys. Res. Lett., 30, 1241, doi:10.1029/ 2002GL016571.

— and Coauthors, 2004: Regions of strong coupling between soil moisture and precipitation. Science, 305, 1138-1140.

, and Coauthors, 2006: GLACE: The Global Land-Atmosphere Coupling Experiment. Part I: Overview. J. Hydrometeor., 7, 590-610. 
Lee, M.-I., S. Schubert, M. J. Suarez, J.-K. E. Schemm, H.-L. Pan, J. Han, and S.-H. Yoo, 2008: Role of convection triggers in the simulation of the diurnal cycle of precipitation over the United States Great Plains in a general circulation model. J. Geophys. Res., 113, D02111, doi:10.1029/2007JD008984.

Lintner, B. R., and J. D. Neelin, 2009: Soil moisture impacts on convective margins. J. Hydrometeor., 10, 1026-1039.

, P. Gentine, K. Findell, F. D'Andrea, A. Sobel, and G. D. Salvucci, 2013: An idealized prototype for large-scale landatmosphere coupling. J. Climate, 26, 2379-2389.

Luo, Y., and Coauthors, 2012: A framework of benchmarking land models. Biogeosci. Discuss., 9, 1899-1944, doi:10.5194/ bgd-9-1899-2012.

Margulis, S. A., and D. Entekhabi, 2001: Feedback between the land surface energy balance and atmospheric boundary layer diagnosed through a model and its adjoint. J. Hydrometeor., 2, 599-620.

Marrel, A., B. Iooss, F. V. Dorpe, and E. Volkova, 2008: An efficient methodology for modeling complex computer codes with Gaussian processes. Comput. Stat. Data Anal., 52, 47314744

_ _ - M. Jullien, B. Laurent, and E. Volkova, 2011: Global sensitivity analysis for models with spatially dependent outputs. Environmetrics, 22, 383-397.

Mei, R., and G. Wang, 2012: Summer land-atmosphere coupling strength in the United States: Comparison among observations, reanalysis data, and numerical models. J. Hydrometeor., 13, 1010-1022.

Mesinger, F., and Coauthors, 2006: North American Regional Reanalysis. Bull. Amer. Meteor. Soc., 87, 343-360.

Milly, P., and K. Dunne, 1994: Sensitivity of the global water cycle to the water-holding capacity of land. J. Climate, 7, 506-526.

Mitchell, K., and Coauthors, 2004: NCEP completes 25-year North American reanalysis: Precipitation assimilation and land surface are two hallmarks. GEWEX News, Vol. 14, International GEWEX Project Office, Silver Spring, MD, 9-12.

Muller, C., L. Back, P. O'Gorman, and K. Emanuel, 2009: A model for the relationship between tropical precipitation and column water vapor. Geophys. Res. Lett., 36, L16804, doi:10.1029/ 2009GL039667.

Notaro, M., 2008: Statistical identification of global hot spots in soil moisture feedbacks among IPCC AR4 models. J. Geophys. Res., 113, D09101, doi:10.1029/2007JD009199.

Pal, J., and E. Eltahir, 2001: Pathways relating soil moisture conditions to future summer rainfall within a model of the landatmosphere system. J. Climate, 14, 1227-1242.

— and - 2003: A feedback mechanism between soil-moisture distribution and storm tracks. Quart. J. Roy. Meteor. Soc., 129, 2279-2297.

Palmer, T., 2012: Towards the probabilistic Earth-system simulator: A vision for the future of climate and weather prediction. Quart. J. Roy. Meteor. Soc., 138B, 841-861, doi:10.1002/qj.1923.

Pan, H., and L. Mahrt, 1987: Interaction between soil hydrology and boundary-layer development. Bound.-Layer Meteor., 38, $185-202$.

Pearl, J., 2009: Causality: Models, Reasoning and Inference. 2nd ed. Cambridge University Press, 484 pp.

Peters, O., and J. Neelin, 2006: Critical phenomena in atmospheric precipitation. Nat. Phys., 2, 393-396, doi:10.1038/nphys314.

Pielke, R. A., R. Avissar, M. Raupach, A. J. Dolman, X. B. Zeng, and A. S. Denning, 1998: Interactions between the atmosphere and terrestrial ecosystems: Influence on weather and climate. Global Change Biol., 4, 461-475.
Robock, A., K. Vinnikov, C. Schlosser, N. Speranskaya, and Y. Xue, 1995: Use of midlatitude soil moisture and meteorological observations to validate soil moisture simulations with biosphere and bucket models. J. Climate, 8, 15-35.

Ruane, A., 2010: NARR's atmospheric water cycle components Part II: Summertime mean and diurnal interactions. J. Hydrometeor., 11, 1220-1233.

Rumelhart, D., G. Hinton, and R. Williams, 1986: Learning internal representations by error propagation. Parallel Distributed Processing: Explorations in the Microstructure of Cognition, D. E. Rumelhart and J. L. McClelland, Eds., MIT Press, 318-362.

Saltelli, A., K. Chan, and E. M. Scott., Eds., 2000: Sensitivity Analysis: Gauging the Worth of Scientific Models. Wiley, 504 pp.

Salvucci, G., 2001: Estimating the moisture dependence of root zone water loss using conditionally averaged precipitation. Water Resour. Res., 37, 1357-1365.

Santanello, J. A., M. A. Friedl, and W. P. Kustas, 2005: An empirical investigation of convective planetary boundary layer evolution and its relationship with the land surface. J. Appl. Meteor., 44, 917-932.

$\longrightarrow,-$ _ and M. Ek, 2007: Convective planetary boundary layer interactions with the land surface at diurnal time scales: Diagnostics and feedbacks. J. Hydrometeor., 8, 1082-1097.

, C. D. Peters-Lidard, S. V. Kumar, C. Alonge, and W.-K. Tao, 2009: A modeling and observational framework for diagnosing local land-atmosphere coupling on diurnal time scales. J. Hydrometeor., 10, 577-599.

,-- , and — 2011: Diagnosing the sensitivity of local landatmosphere coupling via the soil moisture-boundary layer interaction. J. Hydrometeor., 12, 766-786.

Savenije, H., 1995: New definitions for moisture recycling and the relationship with land-use changes in the Sahel. J. Hydrol., 167, 57-78.

Schär, C., and G. Jendritzky, 2004: Climate change: Hot news from summer 2003. Nature, 432, 559-560.

_ D. Lüthi, U. Beyerle, and E. Heise, 1999: The soil-precipitation feedback: A process study with a regional climate model. J. Climate, 12, 722-741.

_ L. Vasilina, F. Pertziger, and S. Dirren, 2004: Seasonal runoff forecasting using precipitation from meteorological data assimilation systems. J. Hydrometeor., 5, 959-973.

Seneviratne, S. I., D. Lüthi, M. Litschi, and C. Schär, 2006: Landatmosphere coupling and climate change in Europe. Nature, 443, 205-209.

— , T. Corti, E. L. Davin, M. Hirschi, E. B. Jaeger, I. Lehner, B. Orlowsky, and A. J. Teuling, 2010: Investigating soil moisture-climate interactions in a changing climate: A review. Earth Sci. Rev., 99, 125-161.

Simpson, T., D. Lin, and W. Chen, 2001a: Sampling strategies for computer experiments: Design and analysis. Int. J. Reliab. Appl., 2 (3), 2009-2240.

_ J. Poplinski, P. N. Kochet, and J. Allen, 2001b: Metamodels for computer-based engineering design: Survey and recommendations. Eng. Comput., 17 (2), 129-150, doi:10.1007/PL00007198.

Storlie, C., L. Swiler, J. Helton, and C. Sallaberry, 2009: Implementation and evaluation of non-parametric regression procedures for sensitivity analysis of computationally demanding models. Reliab. Eng. Syst. Saf., 94 (11), 1735-1763.

Sun, S., and G. Wang, 2012: The complexity of using a feedback parameter to quantify the soil moisture-precipitation relationship. J. Geophys. Res., 117, D11113, doi:10.1029/ 2011JD017173. 
Sutton, C., T. M. Hamill, and T. T. Warner, 2006: Will perturbing soil moisture improve warm-season ensemble forecasts? A proof of concept. Mon. Wea. Rev., 134, 3174-3189.

Tang, B., W. Hsieh, A. Monahan, and F. Tangang, 2000: Skill comparisons between neural networks and canonical correlation analysis in predicting the equatorial Pacific sea surface temperatures. J. Climate, 13, 287-293.

Taylor, C. M., 2008: Intraseasonal land-atmosphere coupling in the West African monsoon. J. Climate, 21, 6636-6648.

- A. Gounou, F. Guichard, P. Harris, R. Ellis, F. Coubreux, and M. De Kauwe, 2011: Frequency of Sahelian storm initiation enhanced over mesoscale soil-moisture patterns. Nat. Geosci., 4, 430-433, doi:10.1038/ngeo1173.

_, R. de Jeu, F. Guichard, P. Harris, and W. Dorigo, 2012: Afternoon rain more likely over drier soils. Nature, 489, 423-426, doi:10.1038/nature11377.

Tuinenburg, O., R. Hutjes, C. Jacobs, and P. Kabat, 2011: Diagnosis of local land-atmosphere feedbacks in India. $J$. Climate, 24, 251-266.

van der Ent, R., and H. Savenije, 2011: Length and time scales of atmospheric moisture recycling, atmospheric chemistry and physics. Atmos. Chem. Phys., 11, 1853-1863, doi:10.5194/ acp-11-1853-2011.

van Herrwaarden, C. C., J. Vilà-Guerau de Arellano, A. F. Moene, and A. A. M. Holtslag, 2009: Interactions between dry-air entrainment, surface evaporation and convective boundarylayer development. Quart. J. Roy. Meteor. Soc., 135, 12771291, doi:10.1002/qj.431.

Volkova, E., B. Iooss, and F. Van Dorpe, 2008: Global sensitivity analysis for a numerical model of radionuclide migration from the RRC "Kurchatrov Institute" radwaste disposal site. Stoch. Environ. Res. Risk Assess., 22, 17-31.

Wei, J., and P. Dirmeyer, 2012: Dissecting soil moistureprecipitation coupling. Geophys. Res. Lett., 39, L19711, doi:10.1029/2012GL053038.

Westra, D., G. Steeneveld, and A. Holtslag, 2012: Some observational evidence for dry soils supporting enhanced high relative humidity at the convection boundary layer top. J. Hydrometeor., 13, 1347-1358.

Zeng, X., M. Barlage, C. Castro, and K. Fling, 2010: Comparison of land-precipitation coupling strength using observations and models. J. Hydrometeor., 11, 979-994. 